\title{
Reliable Fuzzy Control for Active Suspension Systems with Actuator Delay and Fault
}

\author{
Hongyi Li, Honghai Liu, Senior Member, IEEE, Huijun Gao, Senior Member, IEEE \\ and Peng Shi, Senior Member, IEEE
}

\begin{abstract}
This paper is focused on reliable fuzzy $H_{\infty}$ controller design for active suspension systems with actuator delay and fault. Takagi-Sugeno (T-S) fuzzy model approach is adapted in the study with consideration of the sprung and unsprung masses variation, the actuator delay and fault, and other suspension performances. By utilizing parallel-distributed compensation scheme, a reliable fuzzy $H_{\infty}$ performance analysis criterion is derived for the proposed T-S fuzzy model. Then, a reliable fuzzy $H_{\infty}$ controller is designed such that the resulting T-S fuzzy system is reliable in the sense that it is asymptotically stable and has a prescribed $H_{\infty}$ performance under given constraints. The existence condition of the reliable fuzzy $H_{\infty}$ controller is obtained in terms of linear matrix inequalities Finally, a quarter-vehicle suspension model is used to demonstrate the effectiveness and potential of the proposed design techniques.
\end{abstract}

Index Terms $-H_{\infty}$ control, actuator delay, actuator fault, active suspension systems with uncertainty, Fuzzy control.

\section{INTRODUCTION}

$\mathbf{V}$ EHICLE engineering has approved the crucial role of a vehicle suspension playing in evaluating the vehicle dynamics performance. A suspension component has vital functions: for instance, to support the vehicle weight, to provide effective isolation of the chassis from road excitations, to keep tyre contact with the ground, and to maintain the wheels in appropriate position on the road surface. The roles of a vehicle suspension system are to adequately guarantee the stability of the vehicle, while to provide as much comfort as possible for the passengers by serving the basic function of isolating passengers from road-induced vibration and shocks [1]-[4]. Considerable attentions and efforts have been paid to the challenging issue of how to optimize the required

This work was partially supported the 973 Project (2009CB320600973) , the National Science Foundation for Distinguished Young Scholars of P. R. China (60825303), the Foundation for the Author of National Excellent Doctoral Dissertation of China (2007B4), the Key Laboratory of Integrated Automation for the Process Industry (Northeastern University) and a joint $\mathrm{PhD}$ scholarship of University of Portsmouth and Protean Electric Ltd.

$\mathrm{H}$. $\mathrm{Li}$ is with the Research Institute of Intelligent Control and Systems, Harbin Institute of Technology, Harbin 150001, China. H. Li is also with the Intelligent Systems \& Biomedical Robotics Group, School of Creative Technologies, University of Portsmouth, PO5 4BP, UK. Email: hongyi.li@ port.ac.uk

H. Liu is with the Intelligent Systems \& Biomedical Robotics Group, School of Creative Technologies, University of Portsmouth, PO5 4BP, UK Email: honghai.liu@port.ac.uk

H. Gao is with the Research Institute of Intelligent Control and Systems, Harbin Institute of Technology, Harbin 150001, China. Email: hjgao@hit.edu.cn

P. Shi is with the Department of Computing and Mathematical Sciences, University of Glamorgan, Pontypridd, CF37 IDL, U.K. and School of Engineering and Science, Victoria University, Melbourne, Vic 8001, Australia Email: pshi@glam.ac.uk suspension performances, namely, ride comfort, road handling, and suspension deflection. It is evident that many vehicle models and controller design methods have been reported in [5]-[7]. On the other hand, many active suspension control approaches have been presented to handle the tradeoff by utilizing various control techniques such as fuzzy logic and neural network control [8], gain scheduling control [9], linear optimal control [10], adaptive control [11] and $H_{\infty}$ control [2], [12], [13] and their combined methods.

It should be noticed, however, that all the aforementioned suspension control results are under a full reliability assumption that all control components of the systems are in ideal working conditions. Due to the growing complexity of automated control systems, various faults are likely to be encountered, especially faults from actuators and sensors [14] [16]. During the past few decades, many researches have attempted to resolve the reliable and fault tolerant control problems for dynamic systems with uncertainty such as actuator and sensor faults, a great number of theoretic results have also been presented [17]-[20]. For instance, the reliable $H_{\infty}$ controller design problem was been investigated at a context of linear systems [21], and a controller was designed ensuring the resulting control system reliable, i.e., guaranteed asymptotic stability and $H_{\infty}$ performance, under the assumption that all control components of sensors and actuators are operational. As a matter of fact, an active suspension system is different from its counterpart of a passive suspension system in that its actuator has capability of adjusting force to meet the criteria of the vehicle dynamics, such as guaranteeing the stability of the vehicle, securing passenger comfort and satisfying the suspension performance.

However, when either the actuator or sensor faults occur in an active suspension system, the conventional controllers can not achieve better performance in comparison with the reliable and fault-tolerant controllers as discussed in [22]. Therefore, it is challenging to design a reliable controller such that the system stability and performance of the active suspension closed-loop system can be tolerated with sensor or actuator faults. Due to the electrical and electromagnetic characteristics of the actuators and transmission of the measurement information, electrohydraulic actuators are preferably employed to track the desired forces in order to avoid input time delays, it is a commonly key factor to degrade the control performances and even cause instability in the control systems. Controller design schemes recently have been presented for linear systems with different types of delays [23]-[25]. There exist two mainstreams of controller design methods involving 
actuator delays. One is to design a controller by using the integrated system model including actuator dynamics [26]; the other is to consider the actuator time delay in the controller design process in order to design a controller that can stabilize the system and guarantee the closed-loop performance in spite of the existence of time delay [27].

An active suspension system has the ability to enhance vehicle dynamics by relaxing external impact such as road surface on vehicle travel comfort. In terms of its control design, uncertainty of vehicle sprung and unsprung masses such as its loading conditions should be taken into account to meet vehicle travel performance criteria. For instance, the polytopic parameter uncertainties was been employed to model the varying vehicle sprung or unsprung masses [2], [28], [29]. The parameter-dependent controllers was proposed for the quarter-car suspension systems with sprung mass variation [29]. The parameter-independent sampled-data $H_{\infty}$ controller design strategy was presented to handle both sprung and unsprung mass variations in a case study of a quarter-car suspension system [2]. The state of the art in suspension control design in these scenarios, however, could not provide feasible performance for uncertain active suspension systems with actuator delay and fault. Clearly, there is a need for a new controller design method which has capability of satisfying the control condition. On the other hand, since fuzzy sets were proposed by Zadeh [30], fuzzy logic control has developed into a conspicuous and successful branch of automation and control theory. The T-S fuzzy model has been proved as an effective theoretical method and practical tool for representing complex nonlinear systems and applications [31]-[34].

T-S fuzzy model based systems are described as a weighted sum of some simple linear subsystems, and thus are easily analyzable, the success on control analysis and synthesis problems have been also demonstrated by various techniques [35]-[37], [37]-[40]. Recently, research has been conducted to challenge the reliability for the continuous-time T-S fuzzy systems [41][44]. However, in the context of vehicle suspension control design, there are few results on reliable fuzzy $H_{\infty}$ controller design for T-S fuzzy systems with both actuator delay and fault. On the other hand, fuzzy controller design had been investigated for suspension systems in the past years, for example, [45]-[47]. In particular, a T-S model-based fuzzy control design approach was presented for electrohydraulic active suspension systems with input constraints [47]. It is evident, however, there are few results on fuzzy $H_{\infty}$ controller design for uncertain active suspension systems with actuator delay and fault.

This paper is concerned with the problem of reliable fuzzy $H_{\infty}$ control for uncertain active suspension systems with actuator delay and fault based on the T-S fuzzy model approach. The vehicle dynamic system is established by the fact that vehicle sprung and unsprung mass variations, the actuator delay and fault have been taken account into the suspension performances. The parallel-distributed compensation (PDC) scheme is, then, used to develop reliable fuzzy $H_{\infty}$ performance analysis condition for the proposed T-S fuzzy system, the reliable fuzzy $H_{\infty}$ controller is designed to guarantee the systems asymptotic stability and $H_{\infty}$ performance, simultane- ously satisfying the constraint performances. Further, the linear matrix inequality (LMI)-based condition of reliable fuzzy $H_{\infty}$ controller design is derived. Finally, the proposed method is evaluated on a quarter-car suspension model. Simulation results demonstrate the designed reliable fuzzy $H_{\infty}$ controller has robust capability of guaranteeing better suspension performance with uncertainty of the sprung and unsprung mass variations, the actuator delay and fault.

The reminder of this paper is organized as follows. The problem to be addressed is formulated in Section 2. Section 3 presents the reliable fuzzy $H_{\infty}$ controller design results and Section 4 provides fuzzy $H_{\infty}$ controller design scheme. Simulation results are provided to evaluate the proposed method in Section 5, finally the paper is concluded in Section 6.

Notation: The notation used throughout the paper is presented. The superscript $T$ stands for matrix transposition. $\mathbb{R}^{n}$ denotes the $n$-dimensional Euclidean space. $\|\cdot\|_{\infty}$ denotes the $H_{\infty}$ norm for matrices. The notation $P>0(\geq 0)$ is used to denote a symmetric and positive definite (semi-definite) matrix. In symmetric block matrices or complex matrix expressions, an asterisk * is employed to represent a term that is readily induced by symmetry and $\operatorname{diag}\{\ldots\}$ stands for a block-diagonal matrix. $\operatorname{sym}(A)$ is used to denote $A+A^{T}$ for simplicity. Matrices, if the dimensions are not explicitly stated, are assumed to be compatible for algebraic operations. The space of square-integrable vector functions over $[0, \infty)$ is denoted by $L_{2}[0, \infty)$, and for $w=\{w(t)\} \in L_{2}[0, \infty)$, its norm is denoted by $\|w\|_{2}=\sqrt{\int_{t=0}^{\infty}|w(t)|^{2} d t}$.

\section{Problem Formulation}

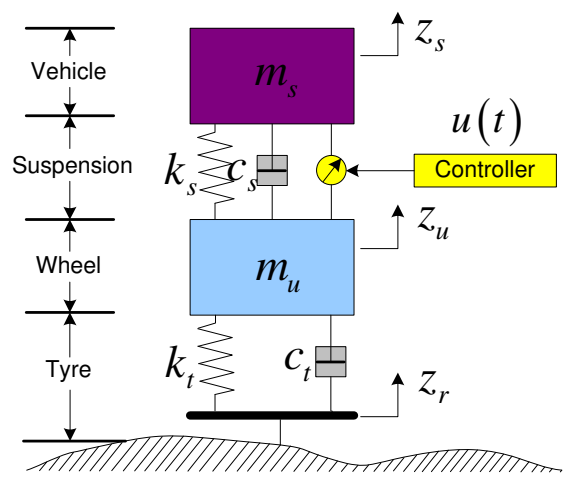

Fig. 1. A quarter-car model

There is a substantially growing interest in investigating the modeling and active control design for active suspension systems in the past three decades. It is due to the fact that these systems play an important role in ensuring the suspension performance, such as ride comfort, road holding, and suspension deflection. A quarter-vehicle model has been used widely in the literature for designing active suspension controller, as shown in Fig. 1, where $m_{s}$ is used to denote the sprung mass, which represents the car chassis; $m_{u}$ is the unsprung mass, which represents mass of the wheel assembly; $u(t)$ stands for the active input of the suspension system; $z_{s}$ and $z_{u}$ denote the displacements of the sprung and unsprung 
masses, respectively; $z_{r}$ is used to denote the road displacement input; $c_{s}$ and $k_{s}$ are damping and stiffness of the suspension system, respectively; $k_{t}$ and $c_{t}$ stand for compressibility and damping of the pneumatic tyre, respectively. Then, the dynamic equation of the suspension model is established as follows:

$$
\begin{aligned}
& m_{u} \ddot{z}_{u}(t)+c_{s}\left[\dot{z}_{u}(t)-\dot{z}_{s}(t)\right]+k_{s}\left[z_{u}(t)-z_{s}(t)\right] \\
& +k_{t}\left[z_{u}(t)-z_{r}(t)\right]+c_{t}\left[\dot{z}_{u}(t)-\dot{z}_{r}(t)\right] \\
= & -u(t), \\
& m_{s} \ddot{z}_{s}(t)+c_{s}\left[\dot{z}_{s}(t)-\dot{z}_{u}(t)\right]+k_{s}\left[z_{s}(t)-z_{u}(t)\right] \\
= & u(t) .
\end{aligned}
$$

Denote $x_{1}(t)=z_{s}(t)-z_{u}(t)$ as the suspension deflection, $x_{2}(t)=z_{u}(t)-z_{r}(t)$ as the tire deflection, $x_{3}(t)=\dot{z}_{s}(t)$ as the sprung mass speed, $x_{4}(t)=\dot{z}_{u}(t)$ as the unsprung mass speed, and $w(t)=\dot{z}_{r}(t)$ as the disturbance input, respectively. The equations in (1) can be rewritten as:

$$
\dot{x}(t)=A(t) x(t)+B_{1}(t) w(t)+B(t) u(t),
$$

where

$$
\begin{aligned}
& A(t)= {\left[\begin{array}{cccc}
0 & 0 & 1 & -1 \\
0 & 0 & 0 & 1 \\
-\frac{k_{s}}{m_{s}} & 0 & -\frac{c_{s}}{m_{s}} & \frac{c_{s}}{m_{s}} \\
\frac{k_{s}}{m_{u}} & -\frac{k_{t}}{m_{u}} & \frac{c_{s}}{m_{u}} & -\frac{c_{s}+c_{t}}{m_{u}}
\end{array}\right], } \\
& B(t)= {\left[\begin{array}{c}
0 \\
0 \\
\frac{1}{m_{s}} \\
-\frac{1}{m_{u}}
\end{array}\right], \quad B_{1}(t)=\left[\begin{array}{c}
0 \\
-1 \\
0 \\
\frac{c_{t}}{m_{u}}
\end{array}\right], } \\
& x(t)=\left[\begin{array}{llll}
x_{1}(t) & x_{2}(t) & x_{3}(t) & x_{4}(t)
\end{array}\right]^{T} .
\end{aligned}
$$

For the control design problems of suspension systems, their performances, such as ride comfort and suspension deflection, road holding are the fundamentals being taken into account. It is widely accepted that ride comfort can be generally quantified by the body acceleration in the vertical direction in the context of a quarter-vehicle model, hence it is practical to choose body acceleration, $\ddot{z}_{s}(t)$, as the first control output. It indicates that one of the objectives is to minimize the vertical acceleration, $\ddot{z}_{s}(t)$, to secure vehicle travel comfort.

Recall $H_{\infty}$ control method, the value of $H_{\infty}$ norm is defined as an upper bound of the root mean square gain, the main objective is to minimize the $H_{\infty}$ norm of the transfer function from the disturbance $w(t)$ to the control output $z_{1}(t)=\ddot{z}_{s}(t)$ with an emphasis on ride comfort improving. Meanwhile, the following required performances have to be taken into account as well:

I) The suspension deflection cannot exceed a maximum value constrained by mechanical structure, that is,

$$
\left|z_{s}(t)-z_{u}(t)\right| \leq z_{\max }
$$

where $z_{\max }$ is the maximum suspension deflection.

II) The dynamic tyre load has to be less than the static tyre load in order to ensure a firm uninterrupted contact of the wheels on the road,

$$
k_{t}\left(z_{u}(t)-z_{r}(t)\right)<\left(m_{s}+m_{u}\right) g .
$$

Based on the above criteria, the body acceleration $\ddot{z}_{s}(t)$ is chosen as the performance control output, the suspension stroke $z_{s}(t)-z_{u}(t)$ and relative dynamic tire load $k_{t}\left(z_{u}(t)-z_{r}(t)\right) /\left(m_{s}+m_{u}\right) g$ are chosen as the second control output $z_{2}(t)$. Therefore, the following system is derived to present the active vehicle suspension system:

$$
\begin{aligned}
\dot{x}(t) & =A(t) x(t)+B_{1}(t) w(t)+B(t) u(t), \\
z_{1}(t) & =C_{1}(t) x(t)+D_{1}(t) u(t), \\
z_{2}(t) & =C_{2}(t) x(t),
\end{aligned}
$$

where $A(t), B_{1}(t)$ and $B(t)$ are defined in (2), and

$$
\begin{aligned}
C_{1}(t) & =\left[\begin{array}{cccc}
-\frac{k_{s}}{m_{s}} & 0 & -\frac{c_{s}}{m_{s}} & \frac{c_{s}}{m_{s}}
\end{array}\right], \quad D_{1}(t)=\frac{1}{m_{s}}, \\
C_{2}(t) & =\left[\begin{array}{cccc}
\frac{1}{z_{\max }} & 0 & 0 & 0 \\
0 & \frac{k_{t}}{\left(m_{s}+m_{u}\right) g} & 0 & 0
\end{array}\right] .
\end{aligned}
$$

Note that the suspension suspension system in (5) is a model with uncertainty in that the sprung mass $m_{s}$ and the unsprung mass $m_{u}$ vary in the given ranges, in which $m_{s}$ and $m_{u}$ denote $m_{s}(t)$ and $m_{u}(t)$ respectively. In the meantime, the actuator delay and fault should be taken into account since the suspension performance could be affected by these factors. It leads to the system as:

$$
\begin{aligned}
\dot{x}(t) & =A(t) x(t)+B_{1}(t) w(t)+B(t) u_{f}(t-d(t)), \\
z_{1}(t) & =C_{1}(t) x(t)+D_{1}(t) u_{f}(t-d(t)) \\
z_{2}(t) & =C_{2}(t) x(t) \\
x(t) & =\phi(t), \quad t \in[-\bar{d}, 0]
\end{aligned}
$$

where $\phi(t)$ is a vector-valued initial continuous function defined on $t \in[-\bar{d}, 0] \cdot d(t)$ denotes the time-varying delay satisfying

$$
0 \leq d(t) \leq \bar{d}, \quad d(t) \leq \mu .
$$

Considering the fault channel from controller to actuator,

$$
u^{f}(t)=m_{a} u(t),
$$

$m_{a}$ is used to represent the possible fault of the corresponding actuator $u^{f}(t) . \check{m}_{a} \leq m_{a} \leq \hat{m}_{a}$, where $\check{m}_{a}$ and $\hat{m}_{a}$ are constant scalars and used to constrain lower and upper bounds of the actuator faults. Three following cases are considered corresponding to three different actuator conditions:

1) $\check{m}_{a}=\hat{m}_{a}=0$, then $m_{a}=0$, which implies that the corresponding actuator $u^{f}(t)$ is completely failed.

2) $\check{m}_{a}=\hat{m}_{a}=1$, thus we obtain $m_{a}=1$, which represents the case of no fault in the actuator $u^{f}(t)$.

3) $0<\breve{m}_{a}<\hat{m}_{a}<1$, which means that there exists partial fault in the corresponding actuator $u^{f}(t)$.

The sprung mass $m_{s}(t)$ and the unsprung mass $m_{u}(t)$ are uncertainties, which vary in a given range, i.e. $m_{s}(t) \in$ $\left[m_{s \min }, m_{s \max }\right]$ and $m_{u}(t) \in\left[m_{u \min }, m_{u \max }\right]$. It is to say that the uncertainty scenarios of the mass $m_{s}(t)$ is bounded by its minimum value $m_{s \min }$ and its maximum value $m_{s \max }$. In addition, the mass $m_{u}(t)$ is bounded by its minimum value $m_{u \text { min }}$ and its maximum value $m_{u \max }$. Next, we obtain the 
values of $\frac{1}{m_{s}(t)}$ and $\frac{1}{m_{u}(t)}$ from $m_{s}(t) \in\left[m_{s \min }, m_{s \max }\right]$ and $m_{u}(t) \in\left[m_{u \min }, m_{u \max }\right]$. Then we have

$$
\begin{aligned}
& \max \frac{1}{m_{s}(t)}=\frac{1}{m_{s \min }}=: \hat{m}_{s}, \quad \min \frac{1}{m_{s}(t)}=\frac{1}{m_{s \max }}=: \check{m}_{s}, \\
& \max \frac{1}{m_{u}(t)}=\frac{1}{m_{u \min }}=: \hat{m}_{u}, \quad \min \frac{1}{m_{u}(t)}=\frac{1}{m_{u \max }}=: \check{m}_{u} .
\end{aligned}
$$

The sector nonlinear method [32] is employed to represent $\frac{1}{m_{s}(t)}$ and $\frac{1}{m_{u}(t)}$ by,

$$
\begin{aligned}
& \frac{1}{m_{s}(t)}=M_{1}\left(\xi_{1}(t)\right) \hat{m}_{s}+M_{2}\left(\xi_{1}(t)\right) \check{m}_{s}, \\
& \frac{1}{m_{u}(t)}=N_{1}\left(\xi_{2}(t)\right) \hat{m}_{u}+N_{2}\left(\xi_{2}(t)\right) \check{m}_{u},
\end{aligned}
$$

where $\xi_{1}(t)=\frac{1}{m_{s}(t)}$ and $\xi_{2}(t)=\frac{1}{m_{u}(t)}$ are premise variables,

$$
\begin{aligned}
M_{1}\left(\xi_{1}(t)\right)+M_{2}\left(\xi_{1}(t)\right) & =1, \\
N_{1}\left(\xi_{2}(t)\right)+N_{2}\left(\xi_{2}(t)\right) & =1 .
\end{aligned}
$$

The membership functions $M_{1}\left(\xi_{1}(t)\right), M_{2}\left(\xi_{1}(t)\right), N_{1}\left(\xi_{2}(t)\right)$ and $N_{2}\left(\xi_{2}(t)\right)$ can be calculated as

$$
\begin{aligned}
M_{1}\left(\xi_{1}(t)\right) & =\frac{\frac{1}{m_{s}(t)}-\check{m}_{s}}{\hat{m}_{s}-\check{m}_{s}}, \quad M_{2}\left(\xi_{1}(t)\right)=\frac{\hat{m}_{s}-\frac{1}{m_{s}(t)}}{\hat{m}_{s}-\check{m}_{s}}, \\
N_{1}\left(\xi_{2}(t)\right) & =\frac{\frac{1}{m_{u}(t)}-\check{m}_{u}}{\hat{m}_{u}-\check{m}_{u}}, \quad N_{2}\left(\xi_{2}(t)\right)=\frac{\hat{m}_{u}-\frac{1}{m_{u}(t)}}{\hat{m}_{u}-\check{m}_{u}} .
\end{aligned}
$$

The member functions are labelled as Heavy, Light, Heavy and Light as shown in Fig.2. Then, the system with uncertainty in (7) is represented by the following fuzzy model:

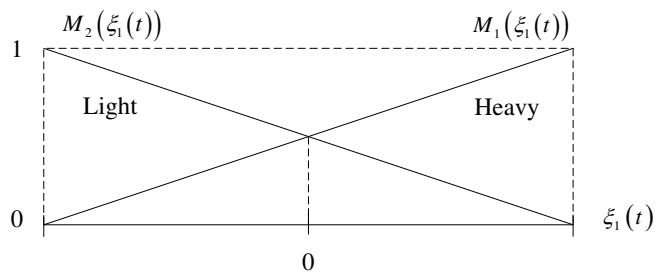

(a)

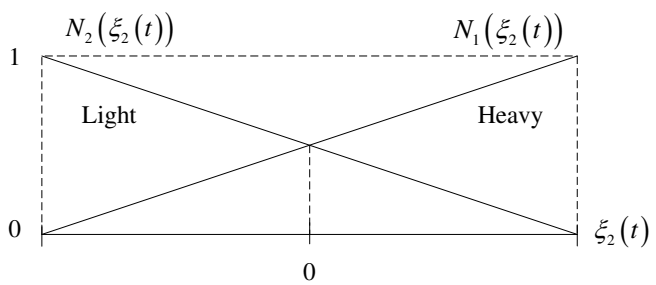

(b)

Fig. 2. (a) Membership functions $M_{1}\left(\xi_{1}(t)\right)$ and $M_{2}\left(\xi_{1}(t)\right)$ (b) Membership functions $N_{1}\left(\xi_{2}(t)\right)$ and $N_{2}\left(\xi_{2}(t)\right)$

Model Rule 1: IF $\xi_{1}(t)$ is Heavy and $\xi_{2}(t)$ is Heavy, THEN

$$
\begin{aligned}
\dot{x}(t) & =A_{1} x(t)+B_{1} u_{f}(t-d(t))+B_{11} w(t), \\
z_{1}(t) & =C_{11} x(t)+D_{11} u_{f}(t-d(t)), \\
z_{2}(t) & =C_{21} x(t),
\end{aligned}
$$

matrices $A_{1}, B_{1}, B_{11}, C_{11}, D_{11}$ and $C_{21}$ are obtained by replacing $\frac{1}{m_{s}(t)}$ and $\frac{1}{m_{u}(t)}$ with matrices $A(t), B(t), B_{1}(t)$, $C_{1}(t), D_{1}(t)$ and $C_{2}(t)$ with $\hat{m}_{s}$ and $\hat{m}_{u}$ respectively.

Model Rule 2: IF $\xi_{1}(t)$ is Heavy and $\xi_{2}(t)$ is Light,

\section{THEN}

$$
\begin{aligned}
\dot{x}(t) & =A_{2} x(t)+B_{2} u_{f}(t-d(t))+B_{12} w(t), \\
z_{1}(t) & =C_{12} x(t)+D_{12} u_{f}(t-d(t)), \\
z_{2}(t) & =C_{22} x(t),
\end{aligned}
$$

matrices $A_{2}, B_{2}, B_{12}, C_{12}, D_{12}$ and $C_{22}$ are obtained by replacing $\frac{1}{m_{s}(t)}$ and $\frac{1}{m_{u}(t)}$ with matrices $A(t), B(t), B_{1}(t)$, $C_{1}(t), D_{1}(t)$ and $C_{2}(t)$ with $\hat{m}_{s}$ and $\check{m}_{u}$ respectively.

Model Rule 3: IF $\xi_{1}(t)$ is Light and $\xi_{2}(t)$ is Heavy,

\section{THEN}

$$
\begin{aligned}
\dot{x}(t) & =A_{3} x(t)+B_{3} u_{f}(t-d(t))+B_{13} w(t), \\
z_{1}(t) & =C_{13} x(t)+D_{13} u_{f}(t-d(t)), \\
z_{2}(t) & =C_{23} x(t),
\end{aligned}
$$

matrices $A_{3}, B_{3}, B_{13}, C_{13}, D_{13}$ and $C_{23}$ are obtained by replacing $\frac{1}{m_{s}(t)}$ and $\frac{1}{m_{u}(t)}$ with matrices $A(t), B(t), B_{1}(t)$, $C_{1}(t), D_{1}(t)$ and $C_{2}(t)$ with $\check{m}_{s}$ and $\hat{m}_{u}$ respectively.

Model Rule 4: IF $\xi_{1}(t)$ is Light and $\xi_{2}(t)$ is Light,

\section{THEN}

$$
\begin{aligned}
\dot{x}(t) & =A_{4} x(t)+B_{4} u_{f}(t-d(t))+B_{14} w(t), \\
z_{1}(t) & =C_{14} x(t)+D_{14} u_{f}(t-d(t)), \\
z_{2}(t) & =C_{24} x(t),
\end{aligned}
$$

matrices $A_{4}, B_{4}, B_{14}, C_{14}, D_{14}$ and $C_{24}$ are obtained by replacing $\frac{1}{m_{s}(t)}$ and $\frac{1}{m_{u}(t)}$ with matrices $A(t), B(t), B_{1}(t)$, $C_{1}(t), D_{1}(t)$ and $C_{2}(t)$ wit $\check{m}_{s}$ and $\check{m}_{u}$ respectively.

Fuzzy blending allows to infer the overall fuzzy model as follows:

$$
\begin{aligned}
\dot{x}(t) & =\sum_{i=1}^{4} h_{i}(\xi(t))\left[A_{i} x(t)+B_{i} u_{f}(t-d(t))+B_{1 i} w(t)\right] \\
z_{1}(t) & =\sum_{i=1}^{4} h_{i}(\xi(t))\left[C_{1 i} x(t)+D_{1 i} u_{f}(t-d(t))\right] \\
z_{2}(t) & =\sum_{i=1}^{4} h_{i}(\xi(t)) C_{2 i} x(t),
\end{aligned}
$$

where

$$
\begin{aligned}
& h_{1}(\xi(t))=M_{1}\left(\xi_{1}(t)\right) \times N_{1}\left(\xi_{2}(t)\right), \\
& h_{2}(\xi(t))=M_{1}\left(\xi_{1}(t)\right) \times N_{2}\left(\xi_{2}(t)\right), \\
& h_{3}(\xi(t))=M_{2}\left(\xi_{1}(t)\right) \times N_{1}\left(\xi_{2}(t)\right), \\
& h_{4}(\xi(t))=M_{2}\left(\xi_{1}(t)\right) \times N_{2}\left(\xi_{2}(t)\right) .
\end{aligned}
$$

It is apparent that the fuzzy weighting functions $h_{i}(\xi(t))$ satisfy $h_{i}(\xi(t)) \geq 0, \quad \sum_{i=1}^{4} h_{i}(\xi(t))=1$. In order to design a fuzzy reliable controllers, PDC is adapted and the following fuzzy controller is obtained:

Control Rule 1: IF $\xi_{1}(t)$ is Heavy and $\xi_{2}(t)$ is Heavy, THEN $u(t)=K_{a 1} x(t)$.

Control Rule 2: IF $\xi_{1}(t)$ is Heavy and $\xi_{2}(t)$ is Light, THEN $u(t)=K_{a 2} x(t)$. 
Control Rule 3: IF $\xi_{1}(t)$ is Light and $\xi_{2}(t)$ is Heavy, THEN $u(t)=K_{a 3} x(t)$.

Control Rule 4: IF $\xi_{1}(t)$ is Light and $\xi_{2}(t)$ is Light, THEN $u(t)=K_{a 4} x(t)$.

Hence, the overall fuzzy control law is represented by

$$
u(t)=\sum_{j=1}^{4} h_{j}(\xi(t)) K_{a j} x(t)
$$

where $K_{a j}(j=1,2,3,4)$ are the local control gains and $u(t-d(t))=\sum_{j=1}^{4} h_{j}(\xi(t-d(t))) K_{a j} x(t-d(t))$. Therefore, in this paper, we assume that $h_{j}(\xi(t-d(t)))$ is well defined for $t \in[-\bar{d}, 0]$, and $h_{j}(\xi(t-d(t))) \geq 0, \quad(j=$ $1,2,3,4) \quad \sum_{j=1}^{4} h_{j}(\xi(t-d(t)))=1$. For simplicity, the following notations will be used:

$$
h_{i}=: h_{i}(\xi(t)), \quad h_{j}^{d}=: h_{j}(\xi(t-d(t))) .
$$

Applying the fuzzy controller (11) to system (10) yields the closed-loop system:

$$
\begin{aligned}
\dot{x}(t)= & \sum_{i=1}^{4} \sum_{j=1}^{4} h_{i} h_{j}^{d}\left[A_{i} x(t)+B_{i} m_{a}(t) K_{a j} x(t-d(t))\right. \\
& \left.+B_{1 i} w(t)\right], \\
z_{1}(t)= & \sum_{i=1}^{4} \sum_{j=1}^{4} h_{i} h_{j}^{d}\left[C_{1 i} x(t)+D_{1 i} m_{a} K_{a j} x(t-d(t))\right], \\
z_{2}(t)= & \sum_{i=1}^{4} h_{i} C_{2 i} x(t) .
\end{aligned}
$$

The T-S fuzzy system in (12) is established based on the practically measurable sprung $m_{s}(t)$ and unsprung $m_{u}(t)$. The sector nonlinearity method [32] is employed to analyze the variation of the sprung $m_{s}(t)$ and unsprung $m_{u}(t)$ and present the T-S fuzzy system in (12).

Without loss of generality, it is assumed, $w \in L_{2}[0, \infty)$, and $\|w\|_{2}^{2} \leq w_{\max }<\infty$. The objective in this subsection is to design the feedback gain matrices $K_{a j}(j=1,2,3,4)$ such that the following requirements are satisfied:

(1) the closed-loop system is asymptotically stable;

(2) under zero initial condition, the closed-loop system guarantees that $\left\|z_{1}\right\|_{2}<\gamma\|w\|_{2}$ for all nonzero $w \in L_{2}[0, \infty)$, where $\gamma>0$ is a prescribed scalar;

(3) the following control output constraints are guaranteed:

$$
\left|\left\{z_{2}(t)\right\}_{q}\right| \leq 1, \quad q=1,2 .
$$

\section{Reliable Fuzzy Controller Design}

In this section, reliable fuzzy $H_{\infty}$ state-feedback controller is derived for the active suspension system with actuator delay and fault. It ensures that the closed-loop system in (12) is asymptotically stable, and it also guarantees a prescribed gain from disturbance $w(t)$ to performance output $z_{1}(t)$, under the condition that the suspension stroke and tire deflection constraints are satisfied. First, the following lemma is presented,

Lemma 1: ( [21]) For a time-varying diagonal matrix $\Phi(t)=\operatorname{diag}\left\{\sigma_{1}(t), \sigma_{2}(t), \cdots, \sigma_{p}(t)\right\}$ and two matrices $R$ and $S$ with appropriate dimensions, if $|\Phi(t)| \leq V$, where $V>0$ is a known diagonal matrix, then for any scalar $\varepsilon>0$, it is true that

$$
R \Phi S+S^{T} \Phi^{T} R^{T} \leq \varepsilon R V R^{T}+\varepsilon^{-1} S^{T} V S .
$$

Next, the following scalars is introduced which will be used in the later development in this paper. $M_{a 0}=\left(\check{m}_{a}+\hat{m}_{a}\right) / 2$, $L_{a}=\left[m_{a}-M_{a 0}\right] / m_{a 0}$ and $J_{a}=\left(\hat{m}_{a}-\check{m}_{a}\right) /\left(\hat{m}_{a}+\check{m}_{a}\right)$. Thus, one has $m_{a}=M_{a 0}\left(I+L_{a}\right)$ and $L_{a}^{T} L_{a} \leq J_{a}^{T} J_{a} \leq I$. Then, it leads to the following theorem.

Theorem 1: Consider the closed-loop system in (12). For given scalars $\bar{d}>0, \mu$ and matrices $K_{a j}$, if there exist matrices $P>0, Q>0, S>0, R>0, N_{j}$, and $M_{j}$ with appropriate dimensions and positive scalars $\varepsilon_{1 i j}>0$ and $\varepsilon_{2 i j}>0(i, j=$ $1,2,3,4)$ such that the following LMIs hold for $q=1,2$ :

$$
\begin{aligned}
& \begin{array}{l}
{\left[\begin{array}{cccccc}
\Phi_{11}^{i j} & \sqrt{\bar{d}} M & \Phi_{13}^{i j} & \Phi_{14}^{i j} & \Phi_{15}^{i j} & \Phi_{16}^{1 i j} \\
0 & -R & 0 & 0 & 0 & 0 \\
0 & 0 & -I & 0 & D_{1 i} & 0 \\
0 & 0 & 0 & -R & \sqrt{\bar{d} R} R B_{i} & 0 \\
0 & 0 & 0 & 0 & -\varepsilon_{1 i j} J_{a}^{-1} & 0 \\
0 & 0 & 0 & 0 & 0 & -\varepsilon_{1 i j} J_{a}^{-1}
\end{array}\right]<0,} \\
{\left[\begin{array}{cccccc}
\Phi_{11}^{i j} & \sqrt{\bar{d}} N & \Phi_{13}^{i j} & \Phi_{14}^{i j} & \Phi_{15}^{i j} & \Phi_{16}^{2 i j} \\
0 & -R & 0 & 0 & 0 & 0 \\
0 & 0 & -I & 0 & D_{1 i} & 0 \\
0 & 0 & 0 & -R & \sqrt{\bar{d} R} R B_{i} & 0 \\
0 & 0 & 0 & 0 & -\varepsilon_{2 i j} J_{a}^{-1} & 0 \\
0 & 0 & 0 & 0 & 0 & -\varepsilon_{2 i j} J_{a}^{-1}
\end{array}\right]<{ }^{2}}
\end{array} \\
& {\left[\begin{array}{cc}
-P & \sqrt{\rho}\left\{C_{2 i}\right\}_{q}^{T} \\
* & -I
\end{array}\right]<0,}
\end{aligned}
$$

where

$$
\begin{aligned}
& \Phi_{11}^{i j}=\Xi_{11}^{i j}+\operatorname{sym}\left(\Xi_{2}\right), \quad \Xi_{11}^{i j}=\left[\begin{array}{cc}
\Theta_{11}^{i j} & \Theta_{12}^{i j} \\
* & -\gamma^{2} I
\end{array}\right], \\
& \Theta_{11}^{i j}=\left[\begin{array}{ccc}
\operatorname{sym}\left(P A_{i}\right)+Q+S & P B_{i} M_{a 0} K_{a j} & 0 \\
* & -(1-\mu) S & 0 \\
* & * & -Q
\end{array}\right] \text {, } \\
& \Theta_{12}^{i j}=\left[\begin{array}{c}
P B_{1 i} \\
0 \\
0
\end{array}\right], \Xi_{2}=\left[\begin{array}{llll}
M & N-M & -N & 0
\end{array}\right] \text {, } \\
& \Phi_{13}^{i j}=\left[\begin{array}{llll}
C_{1 i} & D_{1 i} M_{a 0} K_{a j} & 0 & 0
\end{array}\right]^{T}, \\
& \Phi_{14}^{i j}=\left[\begin{array}{lllll}
\sqrt{\bar{d}} R A_{i} & \sqrt{\bar{d}} R B_{i} M_{a 0} K_{a j} & 0 & \sqrt{\bar{d}} R B_{1 i}
\end{array}\right]^{T}, \\
& \Phi_{15}^{i j}=\left[\begin{array}{cccc}
B_{i}^{T} P & 0 & 0 & 0
\end{array}\right]^{T}, \\
& \Phi_{16}^{1 i j}=\left[\begin{array}{llll}
0 & \varepsilon_{1 i j} M_{a 0} K_{a j} & 0 & 0
\end{array}\right]^{T}, \\
& \Phi_{16}^{2 i j}=\left[\begin{array}{lllll}
0 & \varepsilon_{2 i j} M_{a 0} K_{a j} & 0 & 0
\end{array}\right]^{T}, \\
& M=\left[\begin{array}{llll}
M_{1}^{T} & M_{2}^{T} & M_{3}^{T} & M_{4}^{T}
\end{array}\right]^{T}, \\
& N=\left[\begin{array}{llll}
N_{1}^{T} & N_{2}^{T} & N_{3}^{T} & N_{4}^{T}
\end{array}\right]^{T} .
\end{aligned}
$$

Furthermore,

(1) the closed-loop system is robustly asymptotically stable; 
(2) the performance $\left\|T_{z_{1} w}\right\|_{\infty}<\gamma$ is minimized subject to output constraints (13) with the disturbance energy under the bound $w_{\max }=(\rho-V(0)) / \gamma^{2}$, where $T_{z_{1} w}$ denotes the closedloop transfer function from the road disturbance $w(t)$ to the control output $z_{1}(t)$.

Proof: Considering the Lyapunov-Krasovskii functional as follows:

$$
\begin{aligned}
V(t)= & x^{T}(t) P x(t)+\int_{t-\bar{d}}^{t} x^{T}(s) Q x(s) \mathrm{d} s \\
& +\int_{t-d(t)}^{t} x^{T}(s) S x(s) \mathrm{d} s \\
& +\int_{-\bar{d}}^{0} \int_{t+\alpha}^{t} \dot{x}^{T}(s) R \dot{x}(s) \mathrm{d} s \mathrm{~d} \alpha .
\end{aligned}
$$

The derivative of $V(t)$ along the solution of system (12) is expressed as

$$
\begin{aligned}
\dot{V}(t) \leq & 2 x^{T}(t) P \dot{x}(t)+x^{T}(t)(Q+S) x(t) \\
& -x^{T}(t-\bar{d}) Q x(t-\bar{d})+\bar{d}^{T}(t) S \dot{x}(t) \\
& -(1-\mu) x^{T}(t-d(t)) Q x(t-d(t)) \\
& -\int_{t-d(t)}^{t} \dot{x}^{T}(s) R \dot{x}(s) \mathrm{d} s \\
& -\int_{t-\bar{d}}^{t-d(t)} \dot{x}^{T}(s) R \dot{x}(s) \mathrm{d} s .
\end{aligned}
$$

To develop $H_{\infty}$ performance analysis criterion, the system (12) is stable with $w(t)=0$; then the $H_{\infty}$ performance index is satisfied. For any appropriately dimensioned matrices $\hat{M}$ and $\hat{N}$, the following equalities hold directly according to NewtonLeibniz formula:

$$
\begin{aligned}
\eta_{1}(t)= & 2 \xi^{T}(t) \hat{M} \\
& \times\left(x(t)-x(t-d(t))-\int_{t-d(t)}^{t} \dot{x}(s) \mathrm{d} s\right)=0, \\
\eta_{2}(t)= & 2 \xi^{T}(t) \hat{N} \\
& \times\left(x(t-d(t))-x(t-\bar{d})-\int_{t-\bar{d}}^{t-d(t)} \dot{x}(s) \mathrm{d} s\right)=0,
\end{aligned}
$$

where

$$
\begin{aligned}
\xi^{T}(t) & =\left[\begin{array}{lll}
x^{T}(t) & x^{T}(t-d(t)) & x^{T}(t-\bar{d})
\end{array}\right], \\
\hat{M} & =\left[\begin{array}{lll}
M_{1}^{T} & M_{2}^{T} & M_{3}^{T}
\end{array}\right]^{T}, \hat{N}=\left[\begin{array}{lll}
N_{1}^{T} & N_{2}^{T} & N_{3}^{T}
\end{array}\right]^{T} .
\end{aligned}
$$

Adding $\eta_{1}(t)$ and $\eta_{2}(t)$ into the right hand side of (18), the following inequalities is obtained:

$$
\begin{aligned}
\dot{V}(t) \leq & \sum_{i=1}^{4} \sum_{j=1}^{4} h_{i} h_{j}^{d} \xi^{T}(t)\left[\hat{\Xi}_{i j}+d(t) \hat{M} R^{-1} \hat{M}^{T}\right. \\
& \left.+(\bar{d}-d(t)) \hat{N} R^{-1} \hat{N}^{T}\right] \xi(t) \\
& -\int_{t-d(t)}^{t}\left[\xi^{T}(t) \hat{M}+\dot{x}^{T}(s) R\right] R^{-1} \\
& \times\left[\hat{M}^{T} \xi(t)+R \dot{x}(s)\right] \mathrm{d} s \\
& -\int_{t-\bar{d}}^{t-d(t)}\left[\xi^{T}(t) \hat{N}+\dot{x}^{T}(s) R\right] R^{-1} \\
& \times\left[\hat{N}^{T} \xi(t)+R \dot{x}(s)\right] \mathrm{d} s \\
\leq & \sum_{i=1}^{4} \sum_{j=1}^{4} h_{i} h_{j}^{d} \xi^{T}(t)\left[\hat{\Xi}_{i j}+d(t) \hat{M} R^{-1} \hat{M}^{T}\right. \\
& \left.+(\bar{d}-d(t)) \hat{N} R^{-1} \hat{N}^{T}\right] \xi(t)
\end{aligned}
$$

$$
\begin{gathered}
=\sum_{i=1}^{4} \sum_{j=1}^{4} h_{i} h_{j}^{d} \xi^{T}(t)\left[\frac{d(t)}{\bar{d}}\left(\hat{\Xi}_{i j}+\bar{d} \hat{M} R^{-1} \hat{M}^{T}\right)\right. \\
\left.+\frac{\bar{d}-d(t)}{\bar{d}}\left(\hat{\Xi}_{i j}+\bar{d} \hat{N} R^{-1} \hat{N}^{T}\right)\right] \xi(t),
\end{gathered}
$$

where

$$
\hat{\Xi}_{i j}=\hat{\Theta}_{11}^{i j}+\operatorname{sym}\left(\hat{\Pi}_{2}\right)+\Upsilon \bar{d} R \Upsilon^{T}
$$

and

$$
\hat{\Pi}_{2}=\left[\begin{array}{lll}
\hat{M} & \hat{N}-\hat{M} & -\hat{N}
\end{array}\right], \quad \Upsilon=\left[\begin{array}{lll}
A_{i} & B_{i} m_{a} K_{a j} & 0
\end{array}\right]^{T},
$$

where the matrix $\hat{\Theta}_{11}^{i j}$ is the matrix $\Theta_{11}^{i j}$, where the term $P B_{i} M_{a 0} K_{a j}$ is replaced by $P B_{i} m_{a} K_{a j}$. It is found that

$$
\begin{aligned}
\tilde{\Xi}_{i j}^{1}= & {\left[\begin{array}{ccc}
\hat{\Theta}_{11}^{i j}+\operatorname{sym}\left(\hat{\Pi}_{2}\right) & \sqrt{\bar{d}} \hat{M} & \sqrt{\bar{d}} \Upsilon R \\
* & -R & 0 \\
* & * & -R
\end{array}\right] } \\
\leq & {\left[\begin{array}{ccc}
\Theta_{11}^{i j} & \sqrt{\bar{d}} \hat{M} & \tilde{\Phi}_{14}^{i j} \\
* & -R & 0 \\
* & * & -R
\end{array}\right]+\varepsilon_{1 i j}^{-1} \Lambda^{T} J_{a} \Lambda+\varepsilon_{1 i j} \Delta J_{a} \Delta^{T}, } \\
\tilde{\Xi}_{i j}^{2}= & {\left[\begin{array}{ccc}
\hat{\Theta}_{11}^{i j}+\operatorname{sym}\left(\hat{\Pi}_{2}\right) & \sqrt{\bar{d} \hat{N}} & \sqrt{\bar{d}} \Upsilon R \\
* & -R & 0 \\
* & * & -R
\end{array}\right] } \\
\leq & {\left[\begin{array}{ccc}
\Theta_{11}^{i j} & \sqrt{\bar{d}} \hat{N} & \tilde{\Phi}_{14}^{i j} \\
* & -R & 0 \\
* & * & -R
\end{array}\right]+\varepsilon_{2 i j}^{-1} \Lambda^{T} J_{a} \Lambda+\varepsilon_{2 i j} \Delta J_{a} \Delta^{T}, }
\end{aligned}
$$

and

$$
\begin{aligned}
\tilde{\Phi}_{14}^{i j} & =\left[\begin{array}{llll}
\sqrt{\bar{d}} R A_{i} & \sqrt{\bar{d}} R B_{i} M_{a 0} K_{a j} & 0
\end{array}\right]^{T}, \\
\Lambda & =\left[\begin{array}{lllll}
B_{i}^{T} P & 0 & 0 & \sqrt{\bar{d}} B_{i}^{T} R & 0
\end{array}\right], \\
\Delta^{T} & =\left[\begin{array}{lllll}
0 & M_{a 0} K_{a j} & 0 & 0 & 0
\end{array}\right] .
\end{aligned}
$$

From (14)-(15) and according to Schur complement, $\tilde{\Xi}_{i j}^{1}<0$ and $\tilde{\Xi}_{i j}^{2}<0$ are obtained, it is to say that

$$
\hat{\Xi}_{i j}+\bar{d} \hat{M} R^{-1} \hat{M}^{T}<0, \quad \hat{\Xi}_{i j}+\bar{d} \hat{N} R^{-1} \hat{N}^{T}<0 .
$$

It leads to $\dot{V}(t)<0$, then the system in (12) is asymptotically stable for the delay $d(t)$ satisfying (8). Next, the $H_{\infty}$ performance of the system in (12) is established under zero initial conditions. Firstly, the Lyapunov functional is defined as shown in (17). It is not difficult to achieve:

$$
\begin{aligned}
& \dot{V}(t)+z_{1}^{T}(t) z_{1}(t)-\gamma^{2} w^{T}(t) w(t) \\
\leq & \sum_{i=1}^{4} \sum_{j=1}^{4} h_{i} h_{j}^{d} \bar{\xi}^{T}(t)\left[\check{\Xi}_{i j}+d(t) M R^{-1} M^{T}\right. \\
& \left.+(\bar{d}-d(t)) N R^{-1} N^{T}\right] \bar{\xi}(t) \\
= & \sum_{i=1}^{4} \sum_{j=1}^{4} h_{i} h_{j}^{d} \bar{\xi}^{T}(t)\left[\frac{d(t)}{\bar{d}}\left(\check{\Xi}_{i j}+\bar{d} M R^{-1} M^{T}\right)\right. \\
& \left.+\frac{\bar{d}-d(t)}{\bar{d}}\left(\check{\Xi}_{i j}+\bar{d} N R^{-1} N^{T}\right)\right] \bar{\xi}(t),
\end{aligned}
$$

where

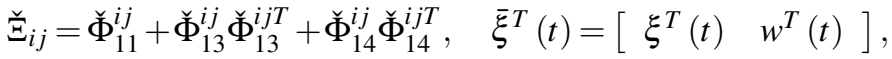


and $\breve{\Phi}_{11}^{i j}, \breve{\Phi}_{13}^{i j}$ and $\breve{\Phi}_{14}^{i j}$ are the matrices $\Phi_{11}^{i j}, \Phi_{13}^{i j}$ and $\Phi_{14}^{i j}$ in which the terms $P B_{i} M_{a 0} K_{a j}, K_{a j}^{T} M_{a 0} D_{1 i}^{T}$ and $\sqrt{\bar{d}} K_{a j}^{T} M_{a 0} B_{i}^{T} R$ are replaced by the terms $P B_{i} m_{a} K_{a j}, K_{a j}^{T} m_{a} D_{1 i}^{T}$ and $K_{a j}^{T} m_{a} B_{i}^{T}$ respectively. According to Schur complement and the above method, we develop

$$
\dot{V}(t)+z_{1}^{T}(t) z_{1}(t)-\gamma^{2} w^{T}(t) w(t)<0,
$$

for all nonzero $w \in L_{2}[0, \infty)$. Under zero initial conditions, we have $V(0)=0$ and $V(\infty) \geq 0$. Integrating both sides of (19) yields $\left\|z_{1}\right\|_{2}<\gamma\|w\|_{2}$ for all nonzero $w \in L_{2}[0, \infty)$, and the $H_{\infty}$ performance is established.

In what follows, we will show that the hard constraints in (13) are guaranteed. Inequality (19) guarantees $\dot{V}(t)-$ $\gamma^{2} w^{T}(t) w(t)<0$. Integrating both sides of the above inequality from zero to any $t>0$, we obtain

$$
V(t)-V(0)<\gamma^{2} \int_{0}^{t} w^{T}(s) w(s) \mathrm{d} s<\gamma^{2}\|w\|_{2}^{2}
$$

From the definition of the Lyapunov functional in (17), we obtain that $x^{T}(t) P x(t)<\rho$ with $\rho=\gamma^{2} w_{\max }+V(0)$. Similar to [2], the following inequality hold

$$
\begin{aligned}
& \max _{t>0}\left|\left\{z_{2}(t)\right\}_{q}\right|^{2} \\
\leq & \max _{t>0}\left\|\sum_{i=1}^{4} h_{i} x^{T}(t)\left\{C_{2 i}\right\}_{q}^{T}\left\{C_{2 i}\right\}_{q} x(t)\right\|_{2} \\
= & \max _{t>0}\left\|\sum_{i=1}^{4} h_{i} x^{T}(t) P^{\frac{1}{2}} P^{-\frac{1}{2}}\left\{C_{2 i}\right\}_{q}^{T}\left\{C_{2 i}\right\}_{q} P^{-\frac{1}{2}} P^{\frac{1}{2}} x(t)\right\|_{2} \\
< & \rho \cdot \theta_{\max }\left(\sum_{i=1}^{4} h_{i} P^{-\frac{1}{2}}\left\{C_{2 i}\right\}_{q}^{T}\left\{C_{2 i}\right\}_{q} P^{-\frac{1}{2}}\right), \quad q=1,2,
\end{aligned}
$$

where $\theta_{\max }(\cdot)$ represents maximal eigenvalue. From the above inequality, it leads to that the constraints in (13) are guaranteed, if

$$
\rho \cdot \sum_{i=1}^{4} h_{i} P^{-\frac{1}{2}}\left\{C_{2 i}\right\}_{q}^{T}\left\{C_{2 i}\right\}_{q} P^{-\frac{1}{2}}<I
$$

which means

$$
\sum_{i=1}^{4} h_{i}\left(\rho \cdot P^{-\frac{1}{2}}\left\{C_{2 i}\right\}_{q}^{T}\left\{C_{2 i}\right\}_{q} P^{-\frac{1}{2}}-I\right)<0,
$$

which is guaranteed by the feasibility of (16). The proof is completed.

Remark 1: In this paper, the free-weight matrices method [48] has been utilized to propose the delay-dependent $H_{\infty}$ performance analysis condition for the time-varying actuator delay $d(t)$. How to develop the less conservative condition is still a challenging research topic. The interval time-varying delay [49] and present less conservative results have been targeted in our future work.

In what follows, the reliable fuzzy $H_{\infty}$ controller existence condition is presented for the active suspension system in (12), it is based on reliable fuzzy $H_{\infty}$ performance analysis criterion in Theorem 1.

Theorem 2: Consider the closed-loop system in (12). For given scalars $\bar{d}>0$ and $\mu$, if there exist matrices $\bar{P}>0, \bar{Q}>0$, $\bar{S}>0, \bar{R}>0, Y_{a j}, \bar{N}_{j}$, and $\bar{M}_{j}$ with appropriate dimensions and positive scalars $\bar{\varepsilon}_{1 i j}>0$ and $\bar{\varepsilon}_{2 i j}>0(i, j=1,2,3,4)$ such that the following LMIs hold for $q=1,2$ :

$$
\begin{aligned}
& {\left[\begin{array}{cccccc}
\bar{\Phi}_{11}^{i j} & \sqrt{\bar{d}} \bar{M} & \bar{\Phi}_{13}^{i j} & \bar{\Phi}_{14}^{i j} & \bar{\Phi}_{15}^{1 i j} & \bar{\Phi}_{16}^{i j} \\
0 & \bar{R}-2 \bar{P} & 0 & 0 & 0 & 0 \\
0 & 0 & -I & 0 & D_{1 i} & 0 \\
0 & 0 & 0 & -R & \bar{\varepsilon}_{1 i j} \sqrt{\bar{d}} B_{i} & 0 \\
0 & 0 & 0 & 0 & -\bar{\varepsilon}_{1 i j} J_{a}^{-1} & 0 \\
0 & 0 & 0 & 0 & 0 & -\bar{\varepsilon}_{1 i j} J_{a}^{-1}
\end{array}\right]<0,} \\
& {\left[\begin{array}{cccccc}
\bar{\Phi}_{11}^{i j} & \sqrt{\bar{d}} \bar{N} & \bar{\Phi}_{13}^{i j} & \bar{\Phi}_{14}^{i j} & \bar{\Phi}_{15}^{2 i j} & \bar{\Phi}_{16}^{i j} \\
0 & \bar{R}-2 \bar{P} & 0 & 0 & 0 & 0 \\
0 & 0 & -I & 0 & D_{1 i} & 0 \\
0 & 0 & 0 & -R & \bar{\varepsilon}_{2 i j} \sqrt{\bar{d}} B_{i} & 0 \\
0 & 0 & 0 & 0 & -\bar{\varepsilon}_{2 i j} J_{a}^{-1} & 0 \\
0 & 0 & 0 & 0 & 0 & -\bar{\varepsilon}_{2 i j} J_{a}^{-1}
\end{array}\right]<0,} \\
& {\left[\begin{array}{cc}
-\bar{P} & \sqrt{\rho} \bar{P}\left\{C_{2 i}\right\}_{q}^{T} \\
* & -I
\end{array}\right]<0,}
\end{aligned}
$$

where

$$
\begin{aligned}
& \bar{\Phi}_{11}^{i j}=\bar{\Xi}_{11}^{i j}+\operatorname{sym}\left(\bar{\Xi}_{2}\right), \quad \bar{\Xi}_{11}^{i j}=\left[\begin{array}{cc}
\bar{\Theta}_{11}^{i j} & \bar{\Theta}_{12}^{i j} \\
* & -\gamma^{2} I
\end{array}\right], \\
& \Theta_{11}^{i j}=\left[\begin{array}{ccc}
\operatorname{sym}\left(A_{i} \bar{P}\right)+\bar{Q}+\bar{S} & B_{i} Y_{a j} & 0 \\
* & -(1-\mu) \bar{S} & 0 \\
* & * & -\bar{Q}
\end{array}\right] \text {, } \\
& \bar{\Theta}_{12}^{i j}=\left[\begin{array}{c}
B_{1 i} \\
0 \\
0
\end{array}\right], \bar{\Xi}_{2}=\left[\begin{array}{llll}
\bar{M} & \bar{N}-\bar{M} & -\bar{N} & 0
\end{array}\right], \\
& \bar{\Phi}_{13}^{i j}=\left[\begin{array}{llll}
C_{1 i} \bar{P} & D_{1 i} Y_{a j} & 0 & 0
\end{array}\right]^{T}, \\
& \bar{\Phi}_{14}^{i j}=\left[\begin{array}{llll}
\sqrt{\bar{d}} A_{i} & \sqrt{\bar{d}} B_{i} Y_{a j} & 0 & \sqrt{\bar{d}} B_{1 i}
\end{array}\right]^{T}, \\
& \bar{\Phi}_{15}^{1 i j}=\left[\begin{array}{llll}
\bar{\varepsilon}_{1 i j} B_{i}^{T} & 0 & 0 & 0
\end{array}\right]^{T}, \\
& \bar{\Phi}_{15}^{2 i j}=\left[\begin{array}{llll}
\bar{\varepsilon}_{2 i j} B_{i}^{T} & 0 & 0 & 0
\end{array}\right]^{T}, \\
& \bar{\Phi}_{16}^{i j}=\left[\begin{array}{llll}
0 & Y_{a j} & 0 & 0
\end{array}\right]^{T}, \\
& \bar{M}=\left[\begin{array}{llll}
\bar{M}_{1}^{T} & \bar{M}_{2}^{T} & \bar{M}_{3}^{T} & \bar{M}_{4}^{T}
\end{array}\right]^{T}, \\
& \bar{N}=\left[\begin{array}{llll}
\bar{N}_{1}^{T} & \bar{N}_{2}^{T} & \bar{N}_{3}^{T} & \bar{N}_{4}^{T}
\end{array}\right]^{T} .
\end{aligned}
$$

Then a reliable controller in the form of (11) exists, such that

(1) the closed-loop system is asymptotically stable;

(2) the performance $\left\|T_{z_{1} w}\right\|_{\infty}<\gamma$ is minimized subject to output constraints (13) with the disturbance energy under the bound $w_{\max }=(\rho-V(0)) / \gamma^{2}$.

Moreover, if inequalities (22)-(24) have a feasible solution, then the control gain $K_{a j}$ in (11) is given by $K_{a j}=M_{a 0}^{-1} Y_{a j} \bar{P}^{-1}$.

Proof: From $(\bar{R}-\bar{P}) \bar{R}^{-1}(\bar{R}-\bar{P}) \geq 0$, we have $-\bar{P} \bar{R}^{-1} \bar{P} \leq$ $\bar{R}-2 \bar{P}$. After replacing $\bar{R}-2 \bar{P}$ in (22)-(23) with $-\bar{P} \bar{R}^{-1} \bar{P}$ and performing corresponding congruence transformation by

$$
\operatorname{diag}\left\{\bar{P}^{-1}, \bar{P}^{-1}, \bar{P}^{-1}, I, \bar{P}^{-1}, I, \bar{R}^{-1}, \bar{\varepsilon}_{1 i j}^{-1} I, \bar{\varepsilon}_{1 i j}^{-1} I\right\},
$$

and by

$$
\operatorname{diag}\left\{\bar{P}^{-1}, \bar{P}^{-1}, \bar{P}^{-1}, I, \bar{P}^{-1}, I, \bar{R}^{-1}, \bar{\varepsilon}_{2 i j}^{-1} I, \bar{\varepsilon}_{2 i j}^{-1} I\right\},
$$


together with the change of matrix variables defined by

$$
\begin{aligned}
P & =\bar{P}^{-1}, \quad R=\bar{R}^{-1}, \quad Q=\bar{P}^{-1} \bar{Q} \bar{P}^{-1}, \\
K_{j} & =M_{a 0}^{-1} Y_{j} \bar{P}^{-1}, \quad S=\bar{P}^{-1} \bar{S} \bar{P}^{-1}, \quad \varepsilon_{1 i j}=\bar{\varepsilon}_{1 i j}^{-1}, \\
\varepsilon_{2} & =\bar{\varepsilon}_{2 i j}^{-1}, \quad M=\operatorname{diag}\left\{\bar{P}^{-1}, \bar{P}^{-1}, \bar{P}^{-1}, I\right\} \bar{M} \bar{P}^{-1} \\
N & =\operatorname{diag}\left\{\bar{P}^{-1}, \bar{P}^{-1}, \bar{P}^{-1}, I\right\} \bar{N} \bar{P}^{-1} .
\end{aligned}
$$

It is concluded that the conditions in (14) and (15) hold. On the other hand, (24) is equivalent to (16) by performing a simple congruence transformation with $\operatorname{diag}\left\{\bar{P}^{-1}, I\right\}$. Therefore, all the conditions in Theorem 1 are satisfied. The proof is completed.

Remark 2: In the study, the conservative will be reduced if the matrices $Q, S, R, \quad M$ and $N$ are replaced by $\quad \sum_{i=1}^{4} h_{i} Q_{i}, \quad \sum_{i=1}^{4} h_{i} S_{i}, \quad \sum_{i=1}^{4} h_{i} R_{i}$, $\sum_{i=1}^{4} h_{i} M_{i}=\sum_{i=1}^{4} h_{i}\left[\begin{array}{llll}M_{1 i}^{T} & M_{2 i}^{T} & M_{3 i}^{T} & M_{4 i}^{T}\end{array}\right]^{T^{T}}$ and $\sum_{i=1}^{4} h_{i} N_{i}=\sum_{i=1}^{4} h_{i}\left[\begin{array}{llll}N_{1 i}^{T} & N_{2 i}^{T} & N_{3 i}^{T} & N_{4 i}^{T}\end{array}\right]^{T}$. However, computation complexion of the existence condition in Theorem 2 of reliable fuzzy $H_{\infty}$ controller design will be increased intensively. Thus, the above proof is employed to handle the tradeoff in this study.

\section{FuZzy $H_{\infty}$ Controller Design}

In the section, fuzzy $H_{\infty}$ controller design is presented for active suspension systems with actuator delay based on T-S fuzzy model method. If there is no actuator fault in the active suspension system, then we obtain,

$$
\begin{aligned}
\dot{x}(t) & =A(t) x(t)+B_{1}(t) w(t)+B(t) u(t-d(t)), \\
z_{1}(t) & =C_{1}(t) x(t)+D_{1}(t) u(t-d(t)), \\
z_{2}(t) & =C_{2}(t) x(t),
\end{aligned}
$$

Based on the above presented fuzzy modeling, the overall fuzzy model is inferred as follows:

$$
\begin{aligned}
\dot{x}(t) & =\sum_{i=1}^{4} h_{i}(\xi(t))\left[A_{i} x(t)+B_{i} u(t-d(t))+B_{1 i} w(t)\right] \\
z_{1}(t) & =\sum_{i=1}^{4} h_{i}(\xi(t))\left[C_{1 i} x(t)+D_{i} u(t-d(t))\right] \\
z_{2}(t) & =\sum_{i=1}^{4} h_{i}(\xi(t)) C_{2 i} x(t) .
\end{aligned}
$$

In addition, the overall fuzzy control law is represented by

$$
u(t)=\sum_{j=1}^{4} h_{j}(\xi(t)) K_{s j} x(t)
$$

For the case of the standard controller (27), the closed-loop system is given by

$$
\begin{aligned}
\dot{x}(t) & =\sum_{i=1}^{4} \sum_{j=1}^{4} h_{i} h_{j}^{d}\left[A_{i} x(t)+B_{i} K_{s j} x(t-d(t))+B_{1 i} w(t)\right] \\
z_{1}(t) & =\sum_{i=1}^{4} \sum_{j=1}^{4} h_{i} h_{j}^{d}\left[C_{1 i} x(t)+D_{1 i} K_{s j} x(t-d(t))\right] \\
z_{2}(t) & =\sum_{i=1}^{4} h_{i} C_{2 i} x(t) .
\end{aligned}
$$

Employing the similar method proposed in the previous section, the following corollary is obtained for the fuzzy $H_{\infty}$ performance analysis at the context of the system in (28) with actuator delay.

Corollary 1: Consider the closed-loop system in (28). Given scalars $\bar{d}>0, \mu$ and matrices $K_{s j}$, if there exist matrices $P>0, Q>0, S>0, R>0, N_{j}$, and $M_{j}(j=1,2,3,4)$ with appropriate dimension such that the following LMIs hold for $q=1,2$ :

$$
\begin{gathered}
{\left[\begin{array}{cccc}
\dot{\Phi}_{11}^{i j} & \sqrt{\bar{d}} M & \dot{\Phi}_{13}^{i j} & \dot{\Phi}_{14}^{i j} \\
0 & -R & 0 & 0 \\
0 & 0 & -I & 0 \\
0 & 0 & 0 & -R
\end{array}\right]<0} \\
{\left[\begin{array}{cccc}
\Phi_{11}^{i j} & \sqrt{\bar{d}} N & \Phi_{13}^{i j} & \dot{\Phi}_{14}^{i j} \\
0 & -R & 0 & 0 \\
0 & 0 & -I & 0 \\
0 & 0 & 0 & -R
\end{array}\right]<0} \\
{\left[\begin{array}{cc}
-P & \sqrt{\rho}\left\{C_{2 i}\right\}_{q}^{T} \\
* & -I
\end{array}\right]<0,}
\end{gathered}
$$

where

$$
\begin{aligned}
\dot{\Phi}_{11}^{i j} & =\dot{\Xi}_{11}^{i j}+\operatorname{sym}\left(\Xi_{2}\right), \Xi_{11}^{i j}=\left[\begin{array}{cc}
\Theta_{11}^{i j} & \Theta_{12}^{i j} \\
* & -\gamma^{2} I
\end{array}\right], \\
\dot{\Phi}_{13}^{i j} & =\left[\begin{array}{cccc}
C_{1 i} & D_{1 i} K_{s j} & 0 & 0
\end{array}\right]^{T}, \\
\dot{\Theta}_{11}^{i j} & =\left[\begin{array}{ccc}
\operatorname{sym}\left(P A_{i}\right)+Q+S & P B_{i} K_{s j} & 0 \\
* & -(1-\mu) S & 0 \\
* & * & -Q
\end{array}\right], \\
\dot{\Phi}_{14}^{i j} & =\left[\begin{array}{cccc}
\sqrt{\bar{d}} R A_{i} & \sqrt{\bar{d}} R B_{i} K_{s j} & 0 & \sqrt{\bar{d}} R B_{1 i}
\end{array}\right]^{T},
\end{aligned}
$$

Take into account the matrices $\Xi_{2}, \Theta_{12}^{i j}, M$ and $N$ in Theorem 1, we obtain,

(1) the closed-loop system is asymptotically stable;

(2) the performance $\left\|T_{z_{1} w}\right\|_{\infty}<\gamma$ is minimized subject to output constraints (13)..

Similarly, the fuzzy $H_{\infty}$ controller design condition as below is derived from Theorem 2.

Corollary 2: Consider the closed-loop system in (28). Given scalars $\bar{d}>0$ and $\mu$, the closed-loop system (12) is asymptotically stable with an $H_{\infty}$ disturbance attenuation level $\gamma$, if there exist matrices $\bar{P}>0, \bar{Q}>0, \bar{S}>0, \bar{R}>0, Y_{s j}, \bar{N}_{j}$, and $\bar{M}_{j}(j=1,2,3,4)$ with appropriate dimensions such that the following LMIs hold for $q=1,2$ :

$$
\begin{aligned}
& {\left[\begin{array}{cccc}
\grave{\Phi}_{11}^{i j} & \bar{\Phi}_{12}^{1 i j} & \grave{\Phi}_{13}^{i j} & \grave{\Phi}_{14}^{i j} \\
0 & \bar{R}-2 \bar{P} & 0 & 0 \\
0 & 0 & -I & 0 \\
0 & 0 & 0 & -R
\end{array}\right]<0} \\
& {\left[\begin{array}{cccc}
\grave{\Phi}_{11}^{i j} & \bar{\Phi}_{12}^{2 i j} & \grave{\Phi}_{13}^{i j} & \grave{\Phi}_{14}^{i j} \\
0 & \bar{R}-2 \bar{P} & 0 & 0 \\
0 & 0 & -I & 0 \\
0 & 0 & 0 & -R
\end{array}\right]<0} \\
& {\left[\begin{array}{cc}
-\bar{P} & \sqrt{\rho} \bar{P}\left\{C_{2 i}\right\}_{q}^{T} \\
* & -I
\end{array}\right]<0,}
\end{aligned}
$$


where

$$
\begin{aligned}
& \grave{\Phi}_{11}^{i j}=\grave{\Xi}_{11}^{i j}+\operatorname{sym}\left(\bar{\Xi}_{2}\right), \quad \grave{\Xi}_{11}^{i j}=\left[\begin{array}{cc}
\grave{\Xi}_{11}^{i j} & \bar{\Theta}_{12}^{i j} \\
* & -\gamma^{2} I
\end{array}\right], \\
& \grave{\Phi}_{13}^{i j}=\left[\begin{array}{llll}
C_{1 i} \bar{P} & D_{1 i} Y_{s j} & 0 & 0
\end{array}\right]^{T}, \\
& \Theta_{11}^{i j}=\left[\begin{array}{ccc}
\operatorname{sym}\left(A_{i} \bar{P}\right)+\bar{Q}+\bar{S} & B_{i} Y_{s j} & 0 \\
* & -(1-\mu) \bar{S} & 0 \\
* & * & -\bar{Q}
\end{array}\right], \\
& \grave{\Phi}_{14}^{i j}=\left[\begin{array}{llll}
\sqrt{\bar{d}} A_{i} & \sqrt{\bar{d}} B_{i} Y_{s j} & 0 & \sqrt{\bar{d}} B_{1 i}
\end{array}\right]^{T},
\end{aligned}
$$

$\bar{\Xi}_{2}, \bar{\Theta}_{12}^{i j}, \bar{M}$ and $\bar{N}$ are defined in Theorem 2 . Then a standard controller in the form of (27) exists, such that

(1) the closed-loop system is asymptotically stable;

(2) the performance $\left\|T_{z_{1} w}\right\|_{\infty}<\gamma$ is minimized subject to output constraint (13).

Moreover, if inequalities (32)-(34) have a feasible solution, then the control gain $K_{s j}$ in (27) is given by $K_{s j}=Y_{s j} \bar{P}^{-1}$.

Remark 3: When the derivative of $d(t)$ is unknown, and the delay $d(t)$ satisfies $0<d(t) \leq \bar{d}$, by setting $S=0$ in (18) and the LMIs-based conditions in Theorems 1-2 and Corollary $1-2$, the reliable fuzzy $H_{\infty}$ controller and fuzzy $H_{\infty}$ controller can be obtained for the systems in (12) and (28) under the condition that the actuator delay $d(t)$ satisfies $0<d(t) \leq \bar{d}$ respectively.

It is can be seen from the LMI-based conditions in Theorem 2 and Corollary 2 both dependent on the matrix variables and the objective scalar $\gamma$, which implies that $\gamma$ can be included as an optimization variable to obtain a lower bound of the guaranteed $H_{\infty}$ performance. Based on the different conditions, reliable fuzzy $H_{\infty}$ controller and fuzzy $H_{\infty}$ controller can be designed with the minimal $\gamma$ by solving the following convex optimization problems:

$$
\begin{aligned}
& \min \gamma \quad \text { s.t. } \quad(22)-(24) . \\
& \left.\bar{P}>\quad 0, \bar{Q}>0, \bar{S}>0, \bar{R}>0, \bar{\varepsilon}_{1 i j}>0, \bar{\varepsilon}_{2 i j}>0, Y_{a j}, \bar{M}, \bar{N} 35\right)
\end{aligned}
$$

and

$$
\begin{aligned}
& \min \gamma \quad \text { s.t. } \quad(32)-(34) . \\
& \bar{P}>\quad 0, \bar{Q}>0, \bar{S}>0, \bar{R}>0, Y_{s j}, \bar{M}, \bar{N} .
\end{aligned}
$$

\section{Simulation Results}

A quarter-vehicle active suspension system is exploited to demonstrate the effectiveness of the proposed approach in this section. The quarter-vehicle suspension model parameters in Table 1 are used for this study. The sprung mass $m_{s}(t)$ is

TABLE I

QUARTER-CAR MODEL PARAMETERS

\begin{tabular}{|c|c|c|c|}
\hline$k_{s}$ & $k_{t}$ & $c_{s}$ & $c_{t}$ \\
\hline $42720 \mathrm{~N} / \mathrm{m}$ & $101115 \mathrm{~N} / \mathrm{m}$ & $1095 \mathrm{Ns} / \mathrm{m}$ & $14.6 \mathrm{Ns} / \mathrm{m}$ \\
\hline
\end{tabular}

assumed to set as the range $[873 \mathrm{~kg}, 1073 \mathrm{~kg}]$ and the unsprung mass $m_{u}(t)$ to $[104 \mathrm{~kg}, 124 \mathrm{~kg}]$. In this study, the maximum allowable suspension stroke is set as $z_{\max }=0.1 \mathrm{~m}$ with $\rho=1$. For the actuator delay $d(t)=5+5 \sin \left(\frac{1}{50}\right)$ ms satisfying $\bar{d}=10$ ms and $\mu=0.1$, we consider fuzzy $H_{\infty}$ controller design for the uncertain active suspension systems in (28). By using the convex optimization in (36), it is found that the minimum guaranteed closed-loop $H_{\infty}$ performance index $\gamma_{\min }$ is 5.3011 and the fuzzy controller gain matrices

$$
K_{s i}=10^{4} \times\left[\begin{array}{llll}
-3.3260 & 5.6998 & -2.5167 & 0.2824
\end{array}\right],
$$

where $i=1,2,3,4$.

It is expected that the desired fuzzy $H_{\infty}$ controller in (27) with the parameters in (37) can be designed such that: 1) the sprung mass acceleration $z_{1}(t)$ is as small as possible; 2 ) the suspension deflection is below the maximum allowable suspension stroke $z_{\max }=0.1 \mathrm{~m}$, which means that $x_{1}(t) / z_{\max }$ below 1 ; 3) the relation dynamic tire load $k_{t} x_{2}(t) /\left(m_{s}(t)+\right.$ $\left.m_{u}(t)\right) g<1$. We first consider the following test road disturbance as

$z_{r}(t)=0.0254 \sin 2 \pi t+0.005 \sin 10.5 \pi t+0.001 \sin 21.5 \pi t(\mathrm{~m})$.

According to [47], the road disturbance has a similar frequency as the car body resonance frequency $(1 \mathrm{~Hz})$ under the condition that high-frequency disturbance added to simulate the rough road surface. In order to carry out the simulation for the fuzzy $H_{\infty}$ controller as in (28), the variational sprung mass $m_{s}(t)$ and the variational unsprung mass $m_{u}(t)$ are set as: $m_{s}(t)=973+100 \sin (t) \mathrm{kg}$ and $m_{u}(t)=114+10 \cos (t)$ $\mathrm{kg}$, for deriving the fuzzy membership functional $h_{i}(\xi(t))$ $(i=1,2,3,4)$. By using the fuzzy $H_{\infty}$ controller in $(27)$ with the parameters in (37), we derive the corresponding closedloop fuzzy system. Fig. 3 depicts the responses of body vertical accelerations and the actuator force for the open- (e.g., passive) and closed-loop (e.g., active) systems. Fig. 4 demonstrates the responses of suspension stroke and tire deflection constraint for both the passive and active systems. It is observed from Fig. 3 that the proposed fuzzy $H_{\infty}$ control strategy reduces the sprung mass acceleration significantly in comparison with the passive suspension under the same road disturbance. The designed fuzzy $H_{\infty}$ controller can achieve the less value of the maximum body acceleration for the active suspension system than the passive system, and passenger acceleration in the active suspension system is reduced significantly, which guarantees better ride comfort. In addition, it can be seen that, from Fig. 4, the suspension deflection constraint $x_{1}(t) / z_{\max }<1$ and the relation dynamic tire load constraint $k_{t} x_{2}(t) /\left(m_{s}(t)+\right.$ $\left.m_{u}(t)\right) g<1$ are guaranteed, which implies the road holding capability is ensured by the desired fuzzy controller. These two figures confirm that the designed standard state-feedback fuzzy $H_{\infty}$ controller can achieve better ride comfort and road handling, guarantee constraint suspension deflection for the active suspension system.

To further evaluate the effectiveness of the proposed fuzzy $H_{\infty}$ controller design strategy with actuator delays, the road disturbance as below is taken into account. In the context of active suspension performance, the road disturbance can be generally assumed as discrete events of relatively short duration and high intensity, caused by, for example, a pronounced bump or pothole on an otherwise smooth road surface. The 


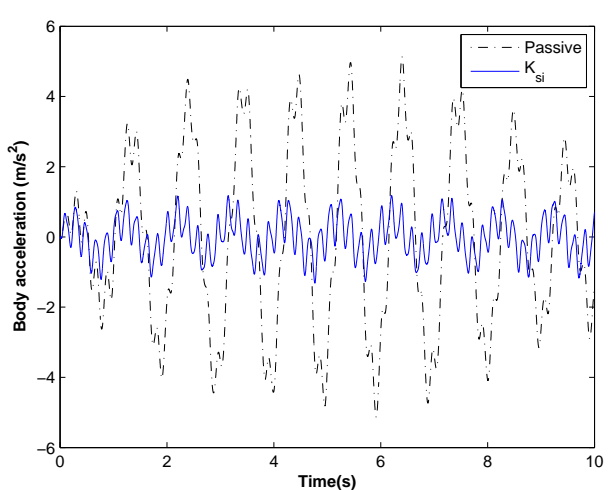

(a)

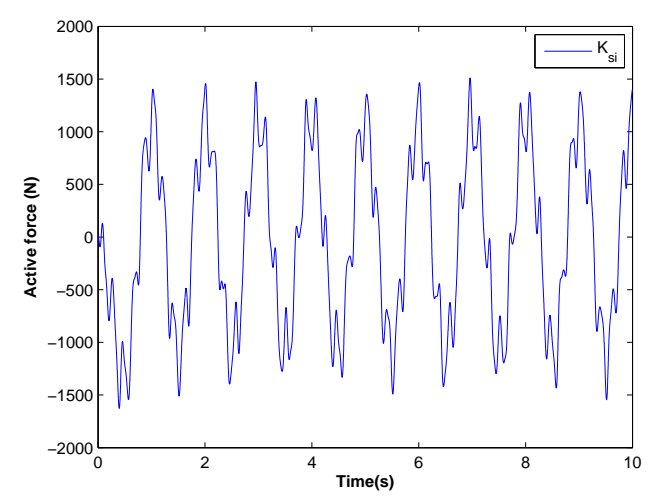

(b)

Fig. 3. (a) Responses of body vertical accelerations, (b) Response of active force.

road surface is represented by,

$$
z_{r}(t)= \begin{cases}\frac{A}{2}\left(1-\cos \left(\frac{2 \pi V}{L} t\right)\right), & \text { if } 0 \leq t \leq \frac{L}{V} \\ 0, & \text { if } t>\frac{L}{V}\end{cases}
$$

where $A$ and $L$ are the height and the length of the bump. Assume $A=50 \mathrm{~mm}, L=6 \mathrm{~m}$ and the vehicle forward velocity as $V=35(\mathrm{~km} / \mathrm{h})$. Fig. 5 illustrates the the responses to body vertical accelerations and the actuator force; Fig. 6 presents the responses to suspension stroke and tire deflection constraint for the passive and active systems under the introduced road disturbance, respectively. The simulation results convincingly demonstrate that the fuzzy $H_{\infty}$ controller offers better suspension performance than the open-loop suspension system.

The effectiveness and advantages of the proposed reliable fuzzy $H_{\infty}$ controller design for active suspension systems with actuator delay and fault will be demonstrated in what follows. The parameters notation in the fuzzy $H_{\infty}$ controller design in the above section is applied here as well. It is assumed that there exists the following actuator fault, namely, $\check{m}_{a}=0.1$, $\hat{m}_{a}=0.5$, which implies $M_{a 0}=0.3$ and $J_{a}=0.2$. Based on the convex optimization presented in (35), we can obtain the minimum guaranteed closed-loop $H_{\infty}$ performance index $\gamma_{\text {min }}$

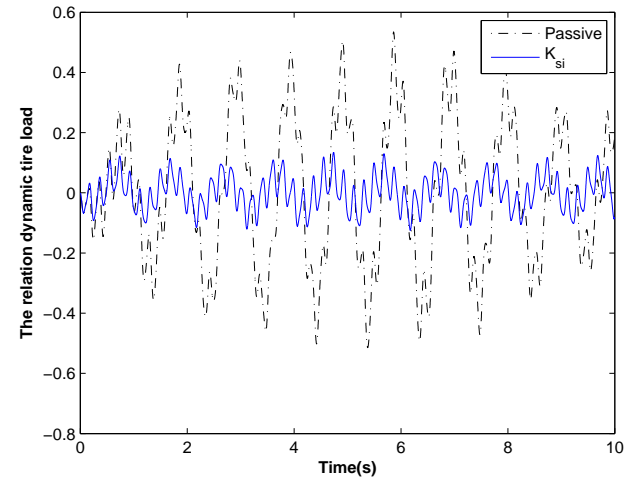

(a)

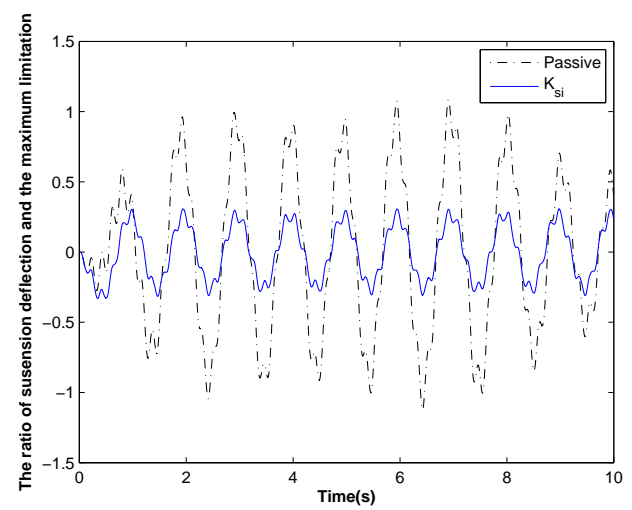

(b)

Fig. 4. (a) Responses of suspension deflection constraint, (b) Responses of tire stroke constraint.

is 28.6991 and the reliable fuzzy controller gain matrices

$$
\begin{aligned}
& K_{a 1}=10^{4} \times\left[\begin{array}{llll}
4.1910 & -0.9700 & -2.5381 & 0.5713
\end{array}\right], \\
& K_{a 2}=10^{4} \times\left[\begin{array}{llll}
4.1916 & -0.9829 & -2.5381 & 0.5711
\end{array}\right], \\
& K_{a 3}=10^{4} \times\left[\begin{array}{llll}
4.1964 & -0.9751 & -2.5382 & 0.5706
\end{array}\right], \\
& K_{a 4}=10^{4} \times\left[\begin{array}{llll}
4.2149 & -0.9439 & -2.5388 & 0.5701
\end{array}\right]
\end{aligned}
$$

For the two kinds of road disturbances, namely, the first case road disturbance as shown in (38) and the second case road disturbance as given in (39). In Figs. 7-10, the responses to the open and closed-loop systems with the actuator delay and fault via the standard fuzzy $H_{\infty}$ controller $K_{s i}$ and reliable controller $K_{a i}(i=1,2,3,4)$ are based on the two different types of road disturbances. These figures show that the less value of the maximum body acceleration is achieved for the active suspension system, the suspension deflection constraint $x_{1}(t) / z_{\max }<1$ is guaranteed and the relation dynamic tire load $k_{t} x_{2}(t) /\left(m_{s}(t)+m_{u}(t)\right) g$ is below 1 in comparison with the passive suspension system, by utilizing the standard fuzzy $H_{\infty}$ controller $K_{s i}$ and reliable controller $K_{a i}(i=1,2,3,4)$ for different three types road disturbances respectively. However, it can be observed from Figs. 7 and 9 that the reliable fuzzy $H_{\infty}$ controller achieves less value of the maximum body acceleration than the standard $H_{\infty}$ controller for the active suspension system with actuator delay and fault. From Fig. 


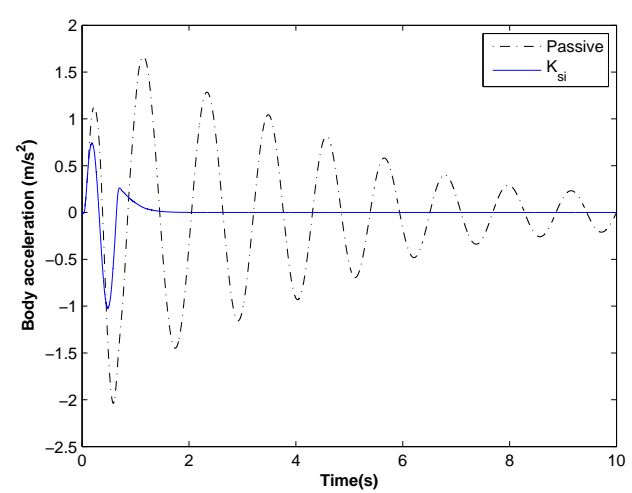

(a)

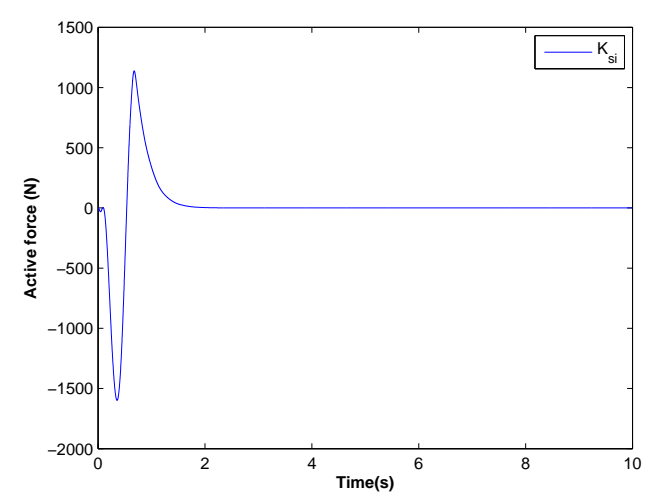

(b)

Fig. 5. (a) Responses of body vertical accelerations, (b) Response of active force.

8 and 10 , it can be seen that $K_{a i}(i=1,2,3,4)$ is capable to provide a much more steady control force in fault condition than conventional controller $K_{s i}(i=1,2,3,4)$.

To further evaluate the suspension system performance under different fuzzy controllers $K_{s i}$ and $K_{a i}(i=1,2,3,4)$, the root mean square (RMS) values of the body acceleration are exploited to demonstrate its advantages. The road disturbances can also be generally assumed as random vibrations, which are consistent and typically specified as random process with a given ground displacement power spectral density (PSD) of

$$
G_{q}(n)=G_{q}\left(n_{0}\right)\left(\frac{n}{n_{0}}\right)^{-c},
$$

where $n_{0}$ denotes the spatial frequency and $n_{0}$ is the reference spatial frequency of $n_{0}=0.1(1 / \mathrm{m}) ; G_{q}\left(n_{0}\right)$ is used to stand for the road roughness coefficient; $c=2$ is the road roughness constant. Related to the time frequency $f$, we have $f=n V$ with $V$ for the vehicle forward velocity. Based on the equation (41), we can obtain the PSD ground displacement:

$$
G_{q}(f)=G_{q}\left(n_{0}\right) n_{0}^{-2} \frac{V}{f^{2}} .
$$

Accordingly, PSD ground velocity is given by

$$
G_{\dot{q}}(f)=(2 \pi f)^{2} G_{q}(f)=4 \pi G_{q}\left(n_{0}\right) n_{0}^{2} V,
$$

which is only related with the vehicle forward velocity. When the vehicle forward velocity is fixed, the ground velocity can

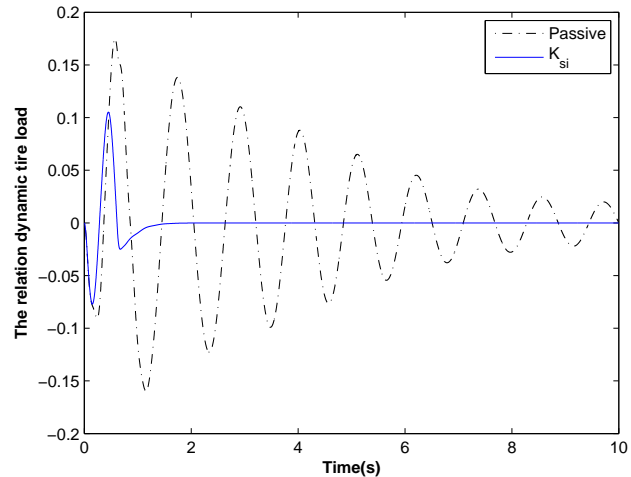

(a)

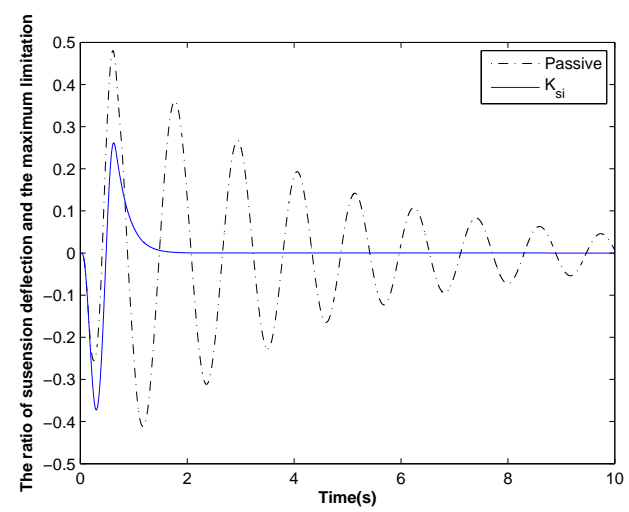

(b)

Fig. 6. (a) Responses of suspension deflection constraint, (b) Responses of tire stroke constraint.

be viewed as a white-noise signal. We choose the four difference road roughness $G_{q}\left(n_{0}\right)=16 \times 10^{-6} \mathrm{~m}^{3}, 64 \times 10^{-6} \mathrm{~m}^{3}$, $256 \times 10^{-6} \mathrm{~m}^{3}$ and $1024 \times 10^{-6} \mathrm{~m}^{3}$, which are corresponded to B Grade (Good), C Grade (Average), D Grade (Poor) and E Grade (Very Poor) for the vehicle forward velocity $V=35$ $(\mathrm{km} / \mathrm{h})$, respectively.

RMS are strictly related to the ride comfort, which are often used to quantify the amount of acceleration transmitted to the vehicle body. The RMS value of variable $x(t)$ is calculated as $\mathrm{RMS}_{x}=\sqrt{(1 / T) \int_{0}^{T} x^{T}(t) x(t) \mathrm{d} t}$. In our study, we choose $T=100 \mathrm{~s}$ to calculate the RMS values of the body acceleration, suspension stroke and relative dynamics tire load for different road roughness coefficient $G_{q}\left(n_{0}\right)$, which are listed in Tables II-IV by using the fuzzy controller $K_{s i}$ and reliable fuzzy controller $K_{a i}$, respectively. It can be observed that these tables indicate that the improvement in ride comfort and the satisfaction of hard constraints can be achieved for the different load conditions by using reliable fuzzy controller $K_{a i}$ compared with the fuzzy controller $K_{s i}$ for the uncertain suspension systems with actuator delay and fault.

\section{CONCLUSIONS}

This paper has investigated the problem of reliable fuzzy $H_{\infty}$ control for active suspension systems with actuator delay and fault. The sprung and unsprung mass variations, the actuator delay and fault, and the suspension performance 
TABLE II

RMS BODY ACCELERATION

\begin{tabular}{||c||c||c||c||}
\hline Parameter & Passive systems & Fuzzy Controller & Reliable Fuzzy Controller \\
\hline$G_{q}\left(n_{0}\right)=16 \times 10^{-6} \mathrm{~m}^{3}$ & 0.0081 & 0.0046 & 0.0041 \\
\hline$G_{q}\left(n_{0}\right)=64 \times 10^{-6} \mathrm{~m}^{3}$ & 0.0152 & 0.0092 & 0.0083 \\
\hline$G_{q}\left(n_{0}\right)=256 \times 10^{-6} \mathrm{~m}^{3}$ & 0.0284 & 0.0183 & 0.0166 \\
\hline$G_{q}\left(n_{0}\right)=1024 \times 10^{-6} \mathrm{~m}^{3}$ & 0.0644 & 0.0387 & 0.0351 \\
\hline
\end{tabular}

TABLE III

RMS SUSPENSION STROKE

\begin{tabular}{||c||c||c||c||}
\hline Parameter & Passive systems & Fuzzy Controller & Reliable Fuzzy Controller \\
\hline$G_{q}\left(n_{0}\right)=16 \times 10^{-6} \mathrm{~m}^{3}$ & $1.7635 \times 10^{-4}$ & $9.7651 \times 10^{-5}$ & $9.5584 \times 10^{-5}$ \\
\hline$G_{q}\left(n_{0}\right)=64 \times 10^{-6} \mathrm{~m}^{3}$ & $3.3536 \times 10^{-4}$ & $1.9626 \times 10^{-4}$ & $1.9057 \times 10^{-4}$ \\
\hline$G_{q}\left(n_{0}\right)=256 \times 10^{-6} \mathrm{~m}^{3}$ & $6.2909 \times 10^{-4}$ & $3.9088 \times 10^{-4}$ & $3.8283 \times 10^{-4}$ \\
\hline$G_{q}\left(n_{0}\right)=1024 \times 10^{-6} \mathrm{~m}^{3}$ & 0.0014 & $8.2616 \times 10^{-4}$ & $8.0992 \times 10^{-4}$ \\
\hline
\end{tabular}

TABLE IV

RMS RELATIVE DYNAMICS TIRE LOAD

\begin{tabular}{||c||c||c||c||}
\hline Parameter & Passive systems & Fuzzy Controller & Reliable Fuzzy Controller \\
\hline$G_{q}\left(n_{0}\right)=16 \times 10^{-6} \mathrm{~m}^{3}$ & $8.3596 \times 10^{-4}$ & $5.2554 \times 10^{-4}$ & $4.9612 \times 10^{-4}$ \\
\hline$G_{q}\left(n_{0}\right)=64 \times 10^{-6} \mathrm{~m}^{3}$ & 0.0016 & 0.0010 & $9.9561 \times 10^{-4}$ \\
\hline$G_{q}\left(n_{0}\right)=256 \times 10^{-6} \mathrm{~m}^{3}$ & 0.0030 & 0.0021 & 0.0020 \\
\hline$G_{q}\left(n_{0}\right)=1024 \times 10^{-6} \mathrm{~m}^{3}$ & 0.0067 & 0.0044 & 0.0042 \\
\hline
\end{tabular}

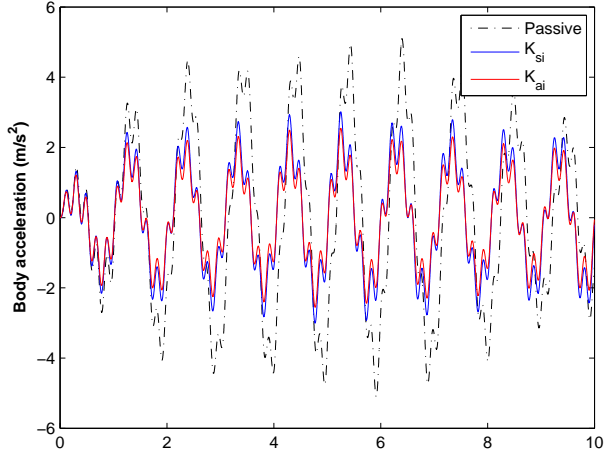

(a)

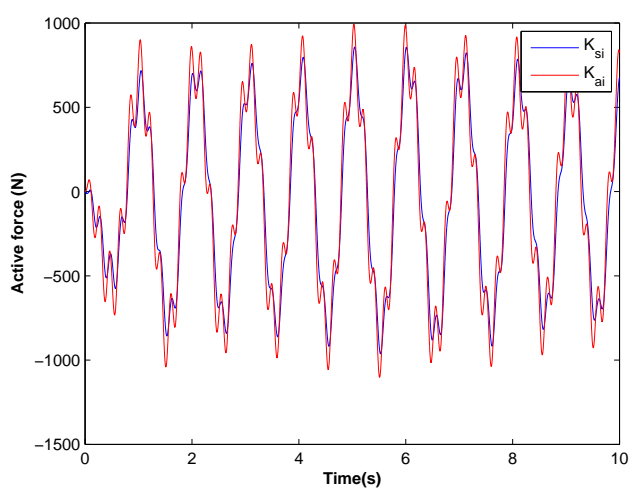

(b)

Fig. 7. (a) Responses of body vertical accelerations, (b) Response of active force.

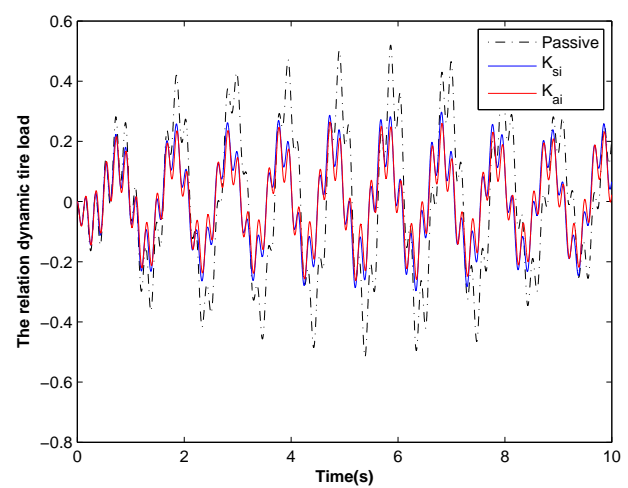

(a)

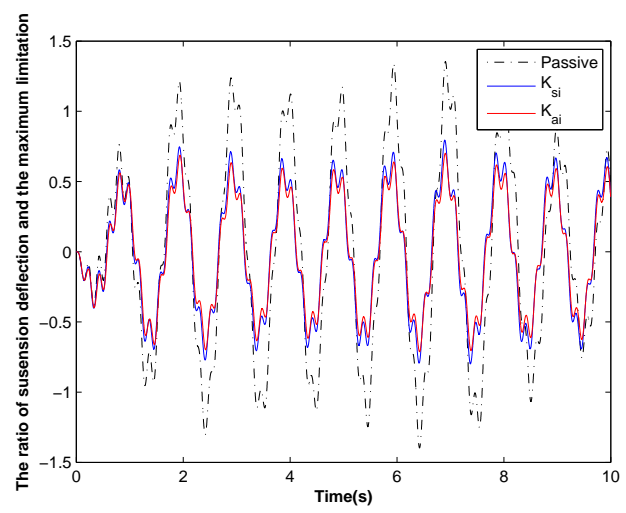

(b)

Fig. 8. (a) Responses of suspension deflection constraint, (b) Responses of tire stroke constraint. 


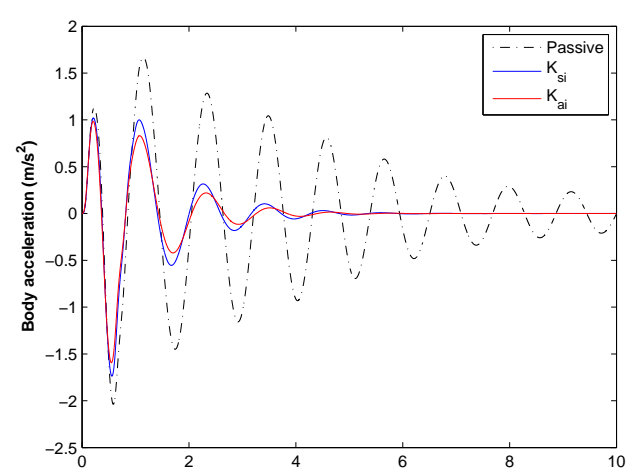

(a)

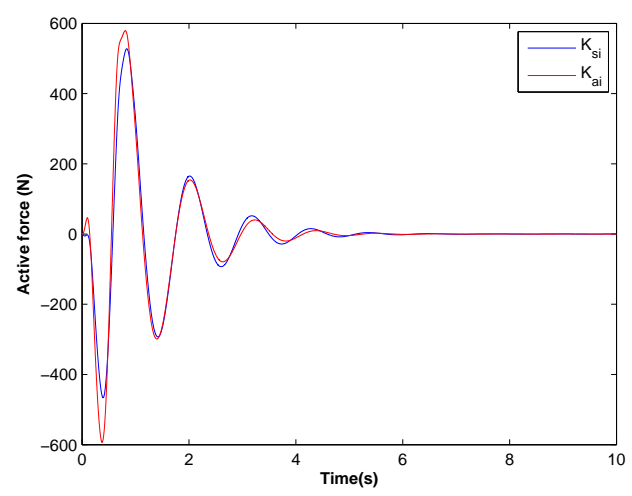

(b)

Fig. 9. (a) Responses of body vertical accelerations, (b) Response of active force.

have all been taken into account to construct the T-S fuzzy system for the control design objective. Based on the PDC scheme and stability theory, the reliable fuzzy $H_{\infty}$ performance analysis condition has been derived for the proposed T-S fuzzy system presenting the active suspension system with uncertainty. Then, the reliable fuzzy $H_{\infty}$ controller has been designed such that the resulting closed-loop T-S fuzzy system is asymptotically stable with $H_{\infty}$ performance, and simultaneously satisfies the constraint suspension performance. A quarter-vehicle suspension model has been used to validate the effectiveness of the proposed design method. Simulation results have clearly demonstrated that the designed reliable fuzzy controller has the capability of guaranteeing a better suspension performance under sprung and unsprung mass variations, actuator delay and fault.

\section{ACKNOWLEDGEMENTS}

The authors are grateful to the Associate Editor and the anonymous referees for their constructive comments and valuable suggestions which have helped improve the quality of the paper.

\section{REFERENCES}

[1] D. Hrovat, "Survey of advanced suspension developments and related optimal control applications," Automatica, vol. 33, no. 10, pp. 1781$1817,1997$.

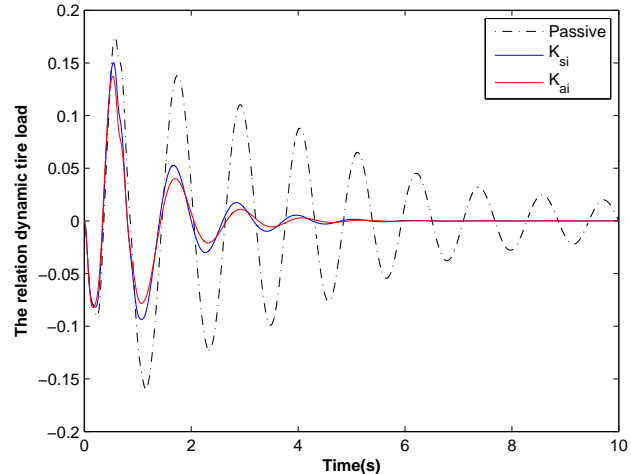

(a)

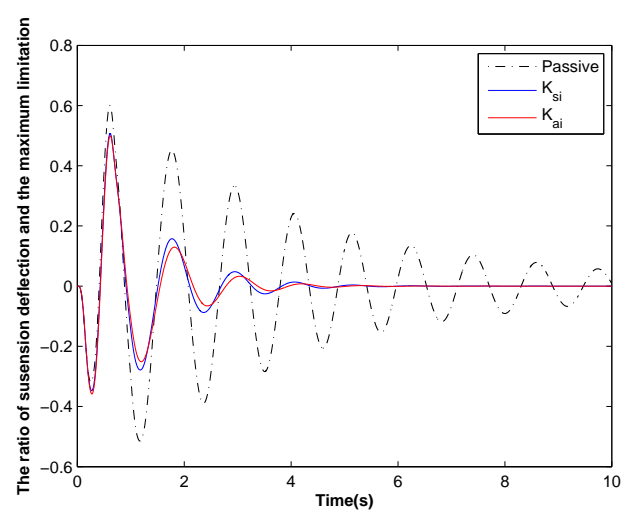

(b)

Fig. 10. (a) Responses of suspension deflection constraint, (b) Responses of tire stroke constraint.

[2] H. Gao, W. Sun, and P. Shi, "Robust sampled-data $H_{\infty}$ control for vehicle active suspension systems ," IEEE Transactions on Control Systems Technology, vol. 18, no. 1, pp. 238-245, 2010.

[3] J. Cao, H. Liu, P. Li, and D. Brown, "State of the art in vehicle active suspension adaptive control systems based on intelligent methodologies," IEEE Transactions on Intelligent Transportation Systems, vol. 9, no. 3, pp. 392-405, 2008.

[4] M. Zapateiro, F. Pozo, H. Karimi, and N. Luo, "Semiactive control methodologies for suspension control with magnetorheological dampers," IEEE/ASME Transactions on Mechatronics,doi: 10.1109 TMECH.2011.2107331.

[5] M. Yamashita, K. Fujimori, K. Hayakawa, and H. Kimura, "Application of $H_{\infty}$ control to active suspension systems," Automatica, vol. 30, no. 11, pp. 1717-1729, 1994.

[6] A. Alleyne and J. Hedrick, "Nonlinear adaptive control of active suspensions," IEEE Transactions on Control Systems Technology, vol. 3, no. 1, pp. 94-101, 1995.

[7] H. Du, K. Yim Sze, and J. Lam, "Semi-active $H_{\infty}$ control of vehicle suspension with magneto-rheological dampers," Journal of Sound and Vibration, vol. 283, no. 3-5, pp. 981-996, 2005.

[8] N. Al-Holou, T. Lahdhiri, D. Joo, J. Weaver, and F. Al-Abbas, "Sliding mode neural network inference fuzzy logic control foractive suspension systems," IEEE Transactions on Fuzzy Systems, vol. 10, no. 2, pp. 234246, 2002.

[9] C. Sivrioglu and I. Cansever, "LPV gain-scheduling controller design for a non-linear quarter-vehicle active suspension system," Transactions of the Institute of Measurement and Control, vol. 31, no. 1, pp. 71-95, 2009.

[10] M. ElMadany and Z. Abduljabbar, "Linear quadratic Gaussian control of a quarter-car suspension," Vehicle System Dynamics, vol. 32, no. 6, pp. 479-497, 1999.

[11] I. Fialho and G. Balas, "Road adaptive active suspension design using linear parameter-varying gain-scheduling," IEEE Transactions on Control Systems Technology, vol. 10, no. 1, pp. 43-54, 2002.

[12] H. Chen and K. Guo, "Constrained $H_{\infty}$ control of active suspensions: 
an LMI approach," IEEE Transactions on Control Systems Technology, vol. 13, no. 3, pp. 412-421, 2005.

[13] H. Karimi, M. Zapateiro, and N. Luo, "A linear matrix inequality approach to $H_{\infty}$ control of vehicle engine-body vibration systems with a time-varying actuator delay," Proceedings of the Institution of Mechanical Engineers, Part I: Journal of Systems and Control Engineering, vol. 222, no. 8, pp. 883-899, 2008.

[14] G. Yang, S. Zhang, J. Lam, and J. Wang, "Reliable control using redundant controllers," IEEE Transactions on Automatic Control, vol. 43, no. 11 , pp. 1588-1593, 2002.

[15] P. Shi, E. Boukas, S. Nguang, and X. Guo, "Robust disturbance attenuation for discrete-time active fault tolerant control systems with uncertainties," Optimal Control Applications and Methods, vol. 24, no. 2, pp. 85-101, 2003.

[16] Y. Guo, B. Jiang, and P. Shi, "Delay-dependent adaptive reconfiguration control in the presence of input saturation and actuator faults," International Journal of Innovative Computing, Information and Control, vol. 6, no. 4, pp. 1873-1882, 2010.

[17] Z. Wang, G. Wei, and G. Feng, "Reliable $H_{\infty}$ control for discrete-time piecewise linear systems with infinite distributed delays," Automatica, vol. 45, no. 12, pp. 2991-2994, 2009.

[18] Z. Mao, B. Jiang, and P. Shi, "Fault-tolerant control for a class of nonlinear sampled-data systems via a Euler approximate observer," Automatica, vol. 46, no. 11, pp. 1852-1859, 2010.

[19] L. Meng and B. Jiang, "Robust active fault-tolerant control for a class of uncertain nonlinear systems with actuator faults," International Journal of Innovative Computing, Information and Control, vol. 6, no. 6, pp. 2637-2644, 2010.

[20] G. Zhou, P. Chen, and T. E., "Reliable control for a class of discretetime state-delayed nonlinear systems with stochastic actuators failures," ICIC Express Letters, vol. 4, no. 6, pp. 2475-2480, 2010.

[21] G. Yang, J. Wang, and Y. Soh, "Reliable $H_{\infty}$ controller design for linear systems," Automatica, vol. 37, no. 5, pp. 717-725, 2001.

[22] Y. Zhao, L. Zhao, and H. Gao, "Vibration control of seat suspension using $H_{\infty}$ reliable control," Journal of Vibration and Control, vol. 16, no. 12 , p. $1859,2010$.

[23] Y. S. Moon, P. G. Park, and W. H. Kwon, "Robust stabilization of uncertain input-delayed systems using reduction method," Automatica, vol. 37, pp. 307-312, 2001.

[24] S. Xu and J. Lam, "Robust $H_{\infty}$ control for uncertain discrete-time-delay fuzzy systems via output feedback controllers," IEEE Transactions on Fuzzy Systems, vol. 13, no. 1, pp. 82-93, 2005.

[25] C. Lin, Z. Wang, and F. Yang, "Observer-based networked control for continuous-time systems with random sensor delays," Automatica, vol. 45, no. 2, pp. 578-584, 2009.

[26] B. Chen, X. Liu, S. Tong, and C. Lin, "Observer-based stabilization of T-S fuzzy systems with input delay," IEEE Transactions on Fuzzy Systems, vol. 16, no. 3, pp. 652-663, 2008.

[27] X. M. Zhang, M. Wu, J. H. She, and Y. He, "Delay-dependent stabilization of linear systems with time-varying state and input delays," Automatica, vol. 41, pp. 1405-1412, 2005.

[28] H. Gao, J. Lam, and C. Wang, "Multi-objective control of vehicle active suspension systems via load-dependent controllers," Journal of Sound and Vibration, vol. 290, no. 3-5, pp. 654-675, 2006.

[29] H. Du, N. Zhang, and J. Lam, "Parameter-dependent input-delayed control of uncertain vehicle suspensions," Journal of Sound and Vibration, vol. 317, no. 3-5, pp. 537-556, 2008.

[30] L. Zadeh, "Fuzzy sets," Information and Control, vol. 8, no. 3, pp. 338353, 1965.

[31] M. Sugeno, "An introductory survey of fuzzy control," Information Sciences, vol. 36, no. 1-2, pp. 59-83, 1985.

[32] K. Tanaka and H. Wang, Fuzzy Control Systems Design and Analysis: A Linear Matrix Inequality Approach. Wiley-Interscience, 2001.

[33] G. Feng, "A survey on analysis and design of model-based fuzzy control systems," IEEE Transactions on Fuzzy Systems, vol. 14, no. 5, pp. 676697, 2006.

[34] C. Lin, G. Wang, and T. Lee, LMI approach to analysis and control of Takagi-Sugeno fuzzy systems with time delay. Springer Verlag, 2007.

[35] H. Lam and M. Narimani, "Quadratic-stability analysis of fuzzy-modelbased control systems using staircase membership functions," IEEE Transactions on Fuzzy Systems, vol. 18, no. 1, pp. 125-137, 2010.

[36] S. Nguang and P. Shi, " $H_{\infty}$ fuzzy output feedback control design for nonlinear systems: an LMI approach," IEEE Transactions on Fuzzy Systems, vol. 11, no. 3, pp. 331-340, 2003.

[37] B. Zhang and S. Xu, "Delay-dependent robust $H_{\infty}$ control for uncertain discrete-time fuzzy systems with time-varying delays," Fuzzy Systems, IEEE Transactions on, vol. 17, no. 4, pp. 809-823, 2009
[38] J. Zhang, P. Shi, and Y. Xia, "Robust adaptive sliding-mode control for fuzzy systems with mismatched uncertainties," IEEE Transactions on Fuzzy Systems, vol. 18, no. 4, pp. 700-711, 2010.

[39] B. Jiang, Z. Mao, and P. Shi, " $H_{\infty}$-filter design for a class of networked control systems via T-S fuzzy-model approach," IEEE Transactions on Fuzzy Systems, vol. 18, no. 1, pp. 201-208, 2010.

[40] H. Dong, Z. Wang, D. Ho, and H. Gao, "Robust $H_{\infty}$ fuzzy outputfeedback control with multiple probabilistic delays and multiple missing measurements," IEEE Transactions on Fuzzy Systems, vol. 18, no. 4, pp. 712-725, 2010

[41] B. Chen and X. Liu, "Reliable control design of fuzzy dynamic systems with time-varying delay," Fuzzy Sets and Systems, vol. 146, no. 3, pp. 349-374, 2004.

[42] H. Wu and H. Zhang, "Reliable $H_{\infty}$ fuzzy control for continuous-time nonlinear systems with actuator failures," IEEE Transactions on Fuzzy Systems, vol. 14, no. 5, p. 609, 2006.

[43] Y. Wang, D. Zhou, and L. Liu, "Reliable memory feedback design for a class of nonlinear fuzzy systems with time-varying delay," International Journal of Automation and Computing, vol. 4, no. 2, pp. 169-176, 2007.

[44] S. Nguang, P. Shi, and S. Ding, "Fault detection for uncertain fuzzy systems: An LMI approach," IEEE Transactions on Fuzzy Systems, vol. 15 , no. 6 , pp. 1251-1262, 2007.

[45] J. Cao, P. Li, and H. Liu, "An interval fuzzy controller for vehicle active suspension systems," IEEE Transactions on Intelligent Transportation Systems, vol. 11, no. 4, pp. 885-895, 2010.

[46] S. Huang and W. Lin, "Adaptive fuzzy controller with sliding surface for vehicle suspension control," IEEE Transactions on Fuzzy Systems, vol. 11 , no. 4 , pp. 550-559, 2003

[47] H. Du and N. Zhang, "Fuzzy control for nonlinear uncertain electrohydraulic active suspensions with input constraint," IEEE Transactions on Fuzzy Systems, vol. 17, no. 2, pp. 343-356, 2009.

[48] Y. He, Q. Wang, C. Lin, and M. Wu, "Delay-range-dependent stability for systems with time-varying delay," Automatica, vol. 43, no. 2, pp. 371-376, 2007.

[49] H. Shao, "New delay-dependent stability criteria for systems with interval delay," Automatica, vol. 45, no. 3, pp. 744-749, 2009.

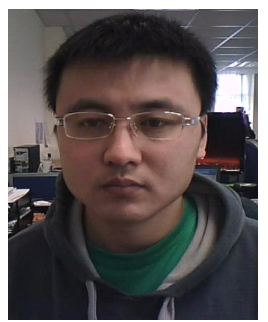

$\mathbf{H}$ ongyi $\mathrm{Li}$ received the B.S. degree and M.S. degrees in Mathematics from Bohai University, Jinzhou, China, in 2006 and 2009, respectively. He is currently pursuing his $\mathrm{PhD}$ degree in the international collaboration programme of the University of Portsmouth, UK, and Harbin Institute of Technology, China. His research interest includes fuzzy control, robust control and their applications in suspension systems.

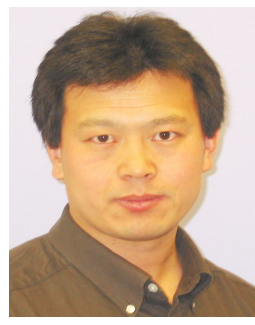

H onghai Liu (M02-SM06) received his Ph.D degree in intelligent robotics from Kings college, University of London, UK, in 2003. He is Professor in Intelligent Systems at the University of Portsmouth, UK. He previously held research appointments at the University of London and University of Aberdeen, $\mathrm{UK}$, and project leader appointments in large-scale industrial control and system integration industry.

$\mathrm{He}$ is interested in approximate computation, pattern recognition, intelligent video analytics, cognitive robotics and their practical applications with an emphasis on approaches which could make contribution to the intelligent connection of perception to action using contextual information. He is a Fellow of IET. 


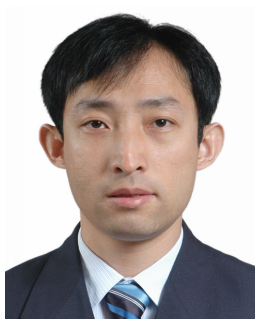

H uijun Gao (SM'09) received the Ph.D. degree in control science and engineering from Harbin Institute of Technology, China, in 2005. He was a Research Associate with the Department of Mechanical Engineering, The University of Hong Kong, from November 2003 to August 2004. From October 2005 to October 2007, he carried out his postdoctoral research with the Department of Electrical and Computer Engineering, University of Alberta, Canada. Since November 2004, he has been with Harbin Institute of Technology, where he is currently a Professor and director of the Research Institute of Intelligent Control and Systems.

Dr Gao's research interests include network-based control, robust control/filter theory, time-delay systems and their engineering applications. He is an Associate Editor for Automatica, IEEE Transactions on Industrial Electronics, IEEE Transactions on Systems Man and Cybernetics Part B: Cybernetics, IEEE Transactions on Fuzzy Systems, IEEE Transactions on Circuits and Systems-I, IEEE Transactions on Control Systems Technology etc.

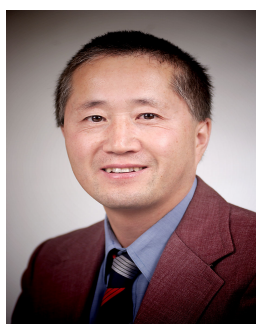

$\mathbf{P}$ eng Shi received the BSc degree in Mathematics from Harbin Institute of Technology, China in 1982; the ME degree in Systems Engineering from Harbin Engineering University, China in 1985; the PhD degree in Electrical Engineering from the University of Newcastle, Australia in 1994; and the PhD degree in Mathematics from the University of South Australia in 1998. He was awarded the degree of Doctor of Science by the University of Glamorgan, UK, in 2006.

Dr Shi was a lecturer in Heilongjiang University, China (1985-1989), in the University of South Australia (1997-1999) and a senior scientist in Defence Science and Technology Organization, Department of Defence, Australia (1999-2005). He joined in the University of Glamorgan, UK, as a professor in 2004. He has also been a professor at Victoria University, Australia since 2008. Dr Shi's research interests include control system design, fault detection techniques, Markov decision processes, and operational research. He has published a number of papers in these areas. In addition, $\mathrm{Dr}$ Shi is a co-author of the three research monographs, Analysis and Synthesis of Systems with Time-Delays (Berlin, Springer, 2009), Fuzzy Control and Filtering Design for Uncertain Fuzzy Systems (Berlin, Springer, 2006), and Methodologies for Control of Jump Time-Delay Systems (Boston, Kluwer, 2003).

Dr Shi currently serves as Editor-in-Chief of Int. J. of Innovative Computing, Information and Control. He is also an Advisory Board Member, Associate Editor, and Editorial Board Member for a number of international journals, including IEEE Transactions on Automatic Control, IEEE Transactions on Systems, Man and Cybernetics-Part B, IEEE Transactions on Fuzzy Systems, Information Sciences, and Int. J. of Systems Science. He is the recipient of the Most Cited Paper Award of Signal Processing in 2009. Dr Shi is a Fellow of the Institute of Engineering and Technology (UK), a Fellow of the Institute of Mathematics and its Applications (UK), and a Senior Member of the Institute of Electrical and Electronic Engineers (USA). 